\title{
HDAC inhibition promotes neuronal outgrowth and counteracts growth cone collapse through CBP/p300 and P/CAF-dependent p53 acetylation
}

\author{
P Gaub ${ }^{1,2}$, A Tedeschi $^{1,2}$, R Puttagunta ${ }^{1}$, T Nguyen $^{1}$, A Schmandke $^{1}$ and S Di Giovanni ${ }^{\star, 1}$
}

\begin{abstract}
Neuronal outgrowth is guided by both extrinsic and intrinsic factors, involving transcriptional regulation. The acetylation of histones and transcription factors, which facilitates promoter accessibility, ultimately promotes transcription, and depends on the balance between histone deacetylases (HDACs) and histone acetyltransferases (HATs) activities. However, a critical function for specific acetylation modifying enzymes in neuronal outgrowth has yet to be investigated. To address this issue, we have used an epigenetic approach to facilitate gene expression in neurons, by using specific HDAC inhibitors. Neurons treated with a combination of HDAC and transcription inhibitors display an acetylation and transcription-dependent increase in outgrowth and a reduction in growth cone collapse on both 'permissive' (poly-D-lysine, PDL) and 'non-permissive' substrates (myelin and chondroitin sulphate proteoglycans (CSPGs)). Next, we specifically show that the expression of the histone acetyltransferases $\mathrm{CBP} / \mathrm{p} 300$ and P/CAF is repressed in neurons by inhibitory substrates, whereas it is triggered by HDAC inhibition on both permissive and inhibitory conditions. Gene silencing and gain of function experiments show that CBP/p300 and P/CAF are key players in neuronal outgrowth, acetylate histone $\mathrm{H3}$ at $\mathrm{K9}-14$ and the transcription factor p53, thereby initiating a pro-neuronal outgrowth transcriptional program. These findings contribute to the growing understanding of transcriptional regulation in neuronal outgrowth and may lay the molecular groundwork for the promotion of axonal regeneration after injury.
\end{abstract}

Cell Death and Differentiation (2010) 17, 1392-1408; doi:10.1038/cdd.2009.216; published online 22 January 2010

Control of transcription has been proven to be important for axonal and dendritic growth during development and for axonal regeneration in the peripheral nervous system. On the contrary, lack of specific gene transcription seems to contribute to the failure in axon re-growth after injury in the central nervous system (CNS). ${ }^{1,2}$

Gene transcription is regulated by epigenetic modifications that contribute to the conformation of chromatin, which allows differential accessibility to promoters. ${ }^{3}$ A permissive or relaxed chromatin conformation (euchromatin) correlates with a relative high level of acetylated histones and is a preferential chromatin state during early embryogenesis, nervous system development and synaptic plasticity. 4,5

The degree of acetylation is the result of a balance between histone acetyltransferases (HATs) and histone deacetylases (HDACs). These two classes of enzymes, respectively, either acetylate or deacetylate lysine residues of histones and various transcription factors (TFs), which form part of the transcription initiation complex. HATs and HDACs are found embedded in large multiprotein complexes within or near euchromatin. ${ }^{6,7}$ HATs modify core histone tails by acetylation of specific lysine residues, and induce a relaxed chromatin conformation, which along with acetylation of a set of TFs promotes DNA accessibility, promoter binding and gene expression. ${ }^{8,9}$ In contrast, HDACs attenuate the transcription process at a particular site by deacetylating these targets, resulting in a more compact chromatin (heterochromatin). The HAT-HDAC system is therefore an essential regulatory switch of gene expression. Enhancement of acetylation can be achieved through the inhibition of deacetylation. This can be accomplished by using several classes of HDAC inhibitors including the hydroxamic acid, trichostatin A (TSA), SAHA and sodium phenyl butyrate $(\mathrm{PB})$, all of which shift the overall balance in favour of HAT activity. ${ }^{7}$

Earlier, increased acetylation of histones and TFs in neurons has been shown to protect from apoptosis in animal models of neurodegeneration ${ }^{10}$ and to promote neuronal differentiation, ${ }^{11-13}$ whose molecular mechanisms are partially shared during neurite/axonal outgrowth. Among several HATs, the CREB-binding protein/p300 (CBP/p300) and the p300-CBPassociated factor (P/CAF) have been associated with the control of neuronal fate. ${ }^{14-16}$ They acetylate histones and TFs that confer protection from neuronal cell death. ${ }^{10,17}$ Recently, we found that neuronal acetylation of p53 at specific lysines in its C-terminus promotes neurite and axonal outgrowth in permissive conditions and is required for axonal regeneration in vivo. ${ }^{18,19}$ However, it is still unknown whether the enhancement of neuronal acetylation and the facilitation of transcription

\footnotetext{
${ }_{1}^{1}$ Laboratory for NeuroRegeneration and Repair, Center for Neurology, Hertie Institute for Clinical Brain Research, University of Tuebingen, Tuebingen, Germany and ${ }^{2}$ Graduate School for Cellular and Molecular Neuroscience, University of Tuebingen, Tuebingen, Germany

*Corresponding author: S Di Giovanni, Laboratory for NeuroRegeneration and Repair, Hertie Institute for Clinical Brain Research, University of Tuebingen, OtfriedMueller Strasse 27, Tuebingen D-72076, Germany. Tel: + 490707129 80449; Fax: + 490707129 4521; E-mail: simone.digiovanni@medizin.uni-tuebingen.de Keywords: acetylation; CBP/p300; P/CAF; p53; neuronal outgrowth

Abbreviations: CGN, cerebellum granular neuron; CBP, CREB-binding protein; CSPG, chondroitin sulphate proteoglycan; GAP-43, growth-associated protein-43; HAT, histone acetyltransferase; HDAC, histone deacetylase; PB, sodium phenyl butyrate; P/CAF, p300/CBP-associated factor; PDL, poly-D-lysine; RCN, rat cortical neuron; SAHA, suberoylanilide hydroxamic acid; TSA, trichostatin A

Received 25.8.09; revised 04.11.09; accepted 30.11.09; Edited by V De Laurenzi; published online 22.1.10
} 
by HDAC inhibition as well as by specific histone acetylating enzymes may promote neuronal outgrowth in physiological conditions and on inhibitory substrates.

We hypothesize that an increase in histone and TF acetylation might have a function in promoting axonal outgrowth by boosting or re-activating the pro-outgrowth transcriptional machinery in neurons in permissive conditions as well as in neurons exposed to outgrowth inhibitory signals such as myelin and proteoglycans.

Here, we specifically show that neuronal hyperacetylation mediated by HDACs inhibition results in transcriptiondependent increased neuronal outgrowth on both permissive and non-permissive substrates, which is accompanied by enhanced promoter acetylation and gene expression of CBP/p300 and P/CAF. Next, gene silencing and overexpression experiments show that neuronal outgrowth on permissive and non-permissive substrates is specifically mediated by CBP/p300 and $\mathrm{P} / \mathrm{CAF}$, whose expression is diminished by myelin signalling. Finally, CBP/p300 and P/CAF together acetylate histones and the TF p53, whose acetylation of specific lysines on its C-terminus by both of these HATs is required for neurite outgrowth and to overcome myelin inhibition.

\section{Results}

Inhibition of deacetylation promotes neuronal outgrowth. Given the requirement for gene expression during neuronal maturation and neurite outgrowth and the decreased outgrowth capacity in mature neurons, we wanted to verify whether this decrease correlated with histone acetylation levels that may affect gene expression. To address this question, we measured the level of histone acetylation in purified cortical and cerebellar neurons at selected developmental stages: E17, P7 and adult (12 weeks). Immunoblotting showed that H3 K9-14 acetylation was clearly elevated in homogenates of freshly dissociated primary neurons at E17 and P7 in comparison to the adult (Figure 1a).

We believe this suggests that the level of histone $\mathrm{H} 3$ acetylation may correlate with the plasticity of neurons and their capacity to develop processes.

Given these findings, we decided to further examine whether increased acetylation could indeed enhance neuronal outgrowth in primary neurons. To this end, we used inhibitors of HDAC I and II such as the hydroxamic acid derivative TSA and PB in primary neurons to enhance the level of overall acetylation and promote neuronal outgrowth.

In initial experiments, we ascertained the toxicity of TSA in primary post-natal P7 cerebellar granule neurons (CGN). CGN were plated on poly-D-lysine (PDL) and stimulated with TSA 1,10 and $100 \mathrm{ng} / \mathrm{ml}$ for 8 or $24 \mathrm{~h}$. We found that TSA at a concentration of $100 \mathrm{ng} / \mathrm{ml}$ resulted in cell death of almost all CGN after both 8 and $24 \mathrm{~h}$ in culture (data not shown).

We then quantified cell viability by counting the number of apoptotic nuclei out of the total number of cells stained with Hoechst in CGN treated with vehicle or TSA at 1 and $10 \mathrm{ng} / \mathrm{ml}$. TSA at a concentration of $10 \mathrm{ng} / \mathrm{ml}$ induced significant cell loss and promoted apoptotic features in surviving neurons (Figure $1 \mathrm{~b}$ ). Whereas, $1 \mathrm{ng} / \mathrm{ml}$ TSA was found to be non-toxic and displayed a minor increase in cell viability (Figure $1 \mathrm{~b}$ ). Given that $1 \mathrm{ng} / \mathrm{ml}$ of TSA does not harm cells, we next examined whether this dose of TSA is sufficient to induce histone acetylation. By immunocytochemistry and immunoblotting for $\mathrm{H} 3$ AcK9-14, we found that TSA at $1 \mathrm{ng} / \mathrm{ml}$ is not only non-toxic, but also sufficient to induce histone hyperacetylation in primary neurons as early as at $4 \mathrm{~h}$ in culture (Figure 1c and $d$ ). This dose regiment is supported by earlier findings in cancer cells ${ }^{20}$ and was used in all the subsequent experiments.

Having established the correct dose of TSA, we next investigated the specific effects of TSA on CGN neurite outgrowth. TSA induced a significant increase in total average neurite length as well as the number of neurites/100 cells (Figure 2a). Hyperacetylation in CGN also significantly increased the rate of non-collapsed growth cones and the growth cone surface area (Figure $2 b$ ).

To examine whether this hyperacetylation-mediated neuronal outgrowth is transcription dependent, we treated cultured CGN with an RNA polymerase II inhibitor, Flavopiridol, ${ }^{21}$ and measured neuronal outgrowth both in the presence and absence of TSA. Results showed that Flavopiridol $(1 \mu \mathrm{M})$ was able to inhibit physiological and TSA-mediated outgrowth by significantly inhibiting the increase in total average neurite length and the number of neurites/100 cells (Figure 2a). In addition, Flavopiridol decreases the percentage of non-collapsed growth cones as well as the growth cone surface area (Figure $2 b$ ). Also, we show by immunoblotting analysis that TSA triggers the expression of the axonal outgrowth marker GAP-43, which is counteracted by Flavopiridol (Figure 2c). Importantly, Flavopiridol at this concentration did not promote neuronal cell death, as measured by the number of surviving cells and the number of cells with fragmented or pyknotic chromatin (data not shown). To verify whether the effects observed with TSA were specific to its function in the inhibition of HDACs I and II, we used another well-characterized HDACs I and II inhibitor, PB. These experiments showed a similar enhancement in histone acetylation and in process outgrowth $24 \mathrm{~h}$ after administration of $0.5 \mu \mathrm{M}$ PB (Supplementary Figure $1 \mathrm{~A}$ and $B$ ). In addition, here we asked whether the well-described enhancement of the pro-outgrowth intrinsic properties of neurons through cAMP would lead to similar neurite outgrowth as HDAC inhibition. We also tested whether the use of another transcriptional inhibitor, Actinomycin D, would block these effects. Indeed, neurons treated with the cAMP analogue dbcAMP showed similar neurite outgrowth as neurons treated with TSA and Actinomycin D inhibited the effect of TSA and dbcAMP dependent outgrowth (Supplementary Figure 1C).

Taken together, these results indicate that hyperacetylation promotes neuronal outgrowth and growth cone remodelling in cultured CGN through inhibition of HDACs I and II by a transcription-dependent mechanism.

Hyperacetylation partially overcomes the inhibitory environment to neuronal outgrowth. So far, we have shown that hyperacetylation promotes neuronal outgrowth through transcriptional modulation and therefore it is likely to enhance promoter accessibility to pro-growth genes. 


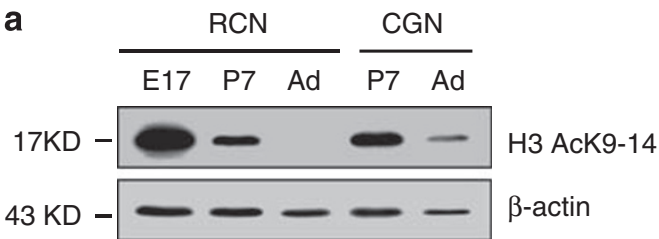

b

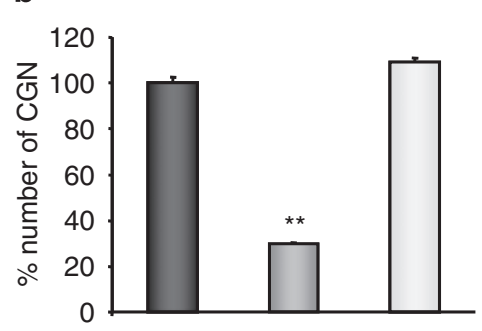

C
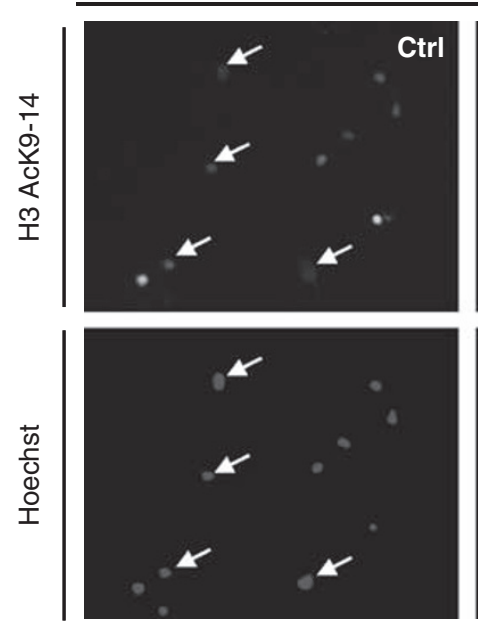

H3 AcK9-14
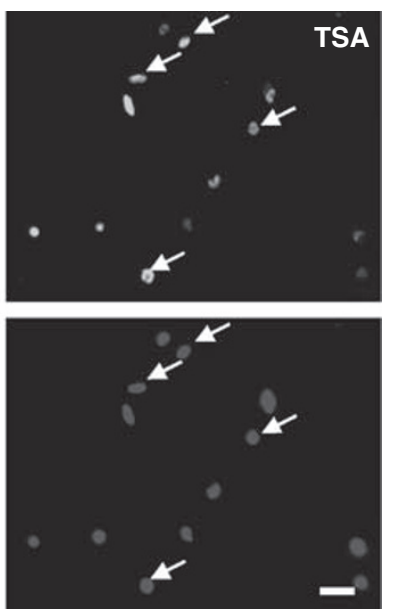

Ctrl

TSA $10 \mathrm{ng} / \mathrm{ml}$

TSA $1 \mathrm{ng} / \mathrm{ml}$

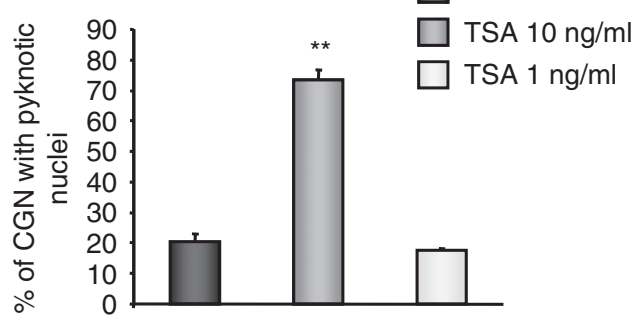

d

TSA

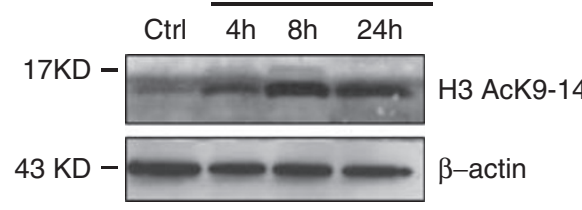

Figure 1 Acetylation of histone $\mathrm{H} 3$ at K9-14 correlates with neuronal maturation and is induced after inhibition of HDAC by TSA. (a) Immunoblotting analysis for H3 AcK9-14 was performed on protein extracts from dissociated RCNs and CGNs at E17, P7 and 12 weeks adult (Ad). A decrease of acetylation with age was observed. $\beta$-actin was used as a loading control. (b) Bar graphs represent the percentage of surviving CGN or CGN with pyknotic nuclei after treatment with $10 \mathrm{ng} / \mathrm{ml}$ or $1 \mathrm{ng} / \mathrm{ml}$ TSA $(24 \mathrm{~h})$ in comparison to vehicle (Ctrl). The result of three independent measurements with an average of 100 cells each is shown. A measure of $10 \mathrm{ng} / \mathrm{ml}$ TSA was found to be toxic $(29.8 \% \pm 2.5$ S.D. of surviving cells TSA versus vehicle, set as $100 \%$ and $73.3 \% \pm 3.5$ pyknotic nuclei TSA versus $20.4 \% \pm 2.8$ vehicle), whereas $1 \mathrm{ng} / \mathrm{ml}$ is comparable to vehicle and considered non-toxic ( $9.2 \% \pm 1.8$ cell survival TSA increase versus vehicle and $17.5 \% \pm 0.7$ pyknotic nuclei TSA versus $20.4 \% \pm 2.4$ vehicle). Asterisks: unpaired two-tailed $t$-test, ${ }^{* *} P$-value $<0.01$. Error bars represent S.D. (c) Immunofluorescence for H3 AcK9-14 in CGN cultured for $24 \mathrm{~h}$ and treated with $1 \mathrm{ng} / \mathrm{ml}$ TSA or vehicle. Arrows document cells that show an increase of staining intensity after TSA treatment. Scale bar: $20 \mu \mathrm{m}$. (d) Immunoblotting analysis for H3 AcK9-14 in CGN cultured for $4 \mathrm{~h}, 8 \mathrm{~h}$ and $24 \mathrm{~h}$ and treated with $1 \mathrm{ng} / \mathrm{ml}$ TSA or vehicle (Ctrl). Shown is a marked increase in the expression of H3 AcK9-K14 after TSA. $\beta$-actin was used as a loading control

Neuronal acetylation at $\mathrm{H} 3 \mathrm{~K} 9-14$ is higher in embryonic and early post-natal than in adult neurons (Figure 1), which are also more sensitive to myelin and proteoglycan inhibition of axon outgrowth as compared with younger neurons. Thus, we next asked whether enhancement of acetylation could protect from neuronal growth cone collapse and outgrowth inhibition on inhibitory substrates such as myelin and chondroitin sulphate proteoglycans (CSPGs). Myelin and CSPGs are in fact known to inhibit outgrowth and induce growth cone collapse, and are important inhibitors of axon outgrowth after CNS injury. ${ }^{22}$

P7 CGN were plated on either myelin or CSPGs with TSA $(1 \mathrm{ng} / \mathrm{ml})$ or vehicle. Outgrowth and growth cone collapse assays were performed at 8 and $24 \mathrm{~h}$ post-plating. Indeed, data analysis showed that TSA significantly overcomes myelin and CSPGs-mediated inhibition of process outgrowth and growth cone collapse (Figure 3a-c, and Supplementary Figure 2). Hyperacetylation induced an increase in total average neurite length as well as an increase in the number of neurites/100 cells on myelin and on CSPGs. In addition, we observed a TSA-dependent increase in the number of non-collapsed growth cones at 8 and $24 \mathrm{~h}$ in culture on myelin and CSPGs, respectively.

Analogously to what was done on permissive substrates, we also assessed whether another HDAC inhibitor such as $\mathrm{PB}$ and the enhancement of CAMP using the analogue dbcAMP would lead to improved neurite outgrowth on myelin. Results show that indeed both PB and dbcAMP promote neurite outgrowth similarly to TSA (Supplementary Figure $3 \mathrm{~A})$. In addition, the transcriptional inhibitors 
a
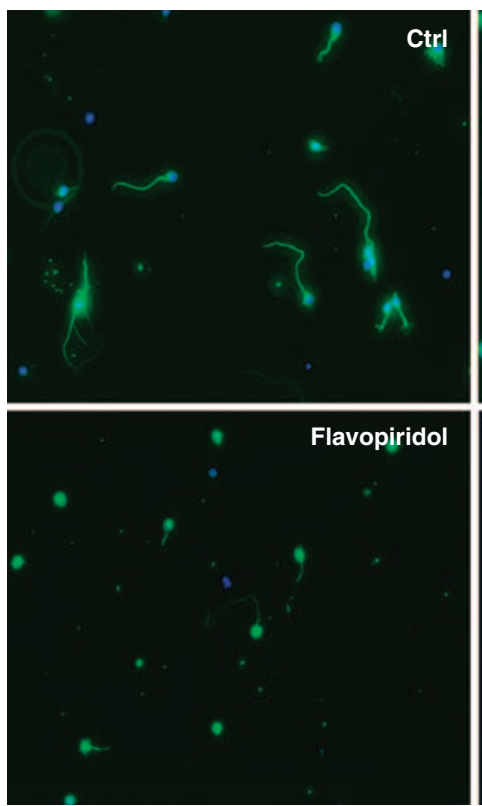

Hoechst

$\beta$ III Tubulin

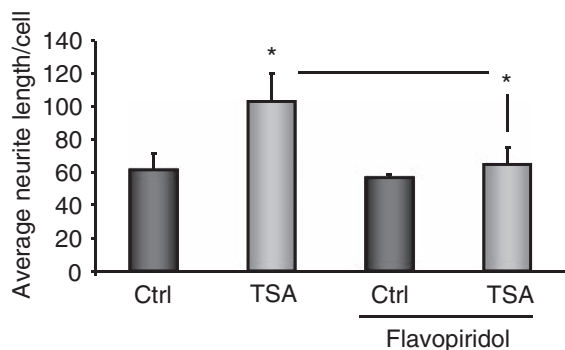

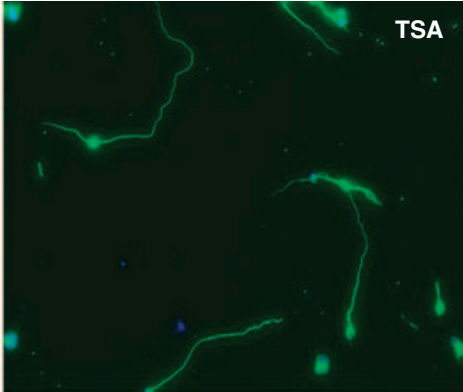
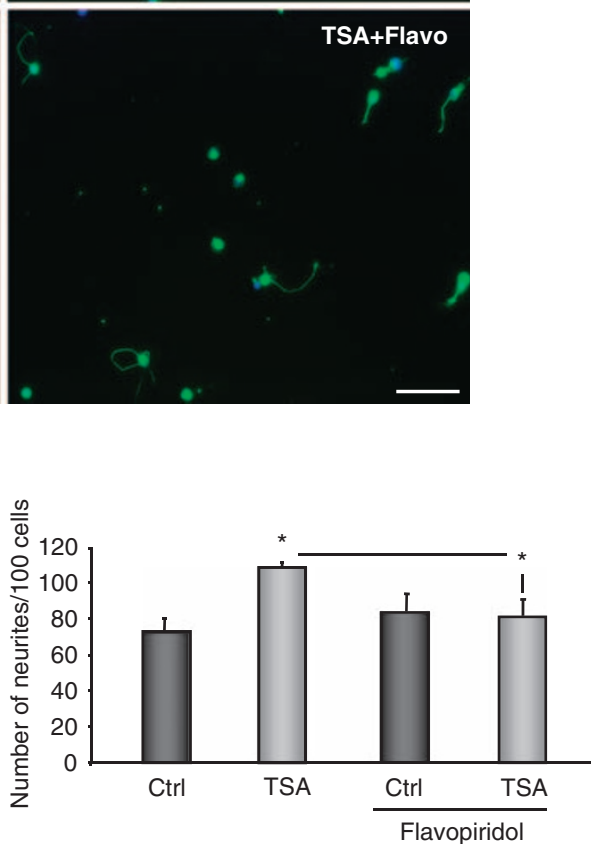

Figure 2 HDAC inhibition promotes neurite outgrowth through increased transcription. (a) Immunofluorescence of CGN shows the effect of $1 \mathrm{ng} / \mathrm{ml}$ TSA and $1 \mu \mathrm{M}$ Flavopiridol after $24 \mathrm{~h}$ treatment in comparison to vehicle (Ctrl). Scale bar: $50 \mu \mathrm{m}$. Bar graphs show quantification of neurite measurements from three independent measurements ( $n$ : 100 cells each), including the average neurite length per cell in $\mu \mathrm{m}$ and number of neurites/100 cells. Neurite measurements show a significant enhancement in neurite length ( $104 \mu \mathrm{m} \pm 16.8$ S.D. TSA versus $61 \mu \mathrm{m} \pm 10.2$ vehicle) and numbers ( $111 \pm 6.5 \mathrm{TSA}$ versus $72 \pm 8$ vehicle) after TSA (1 $\mathrm{ng} / \mathrm{ml})$ in CGN at $24 \mathrm{~h}$. Flavopiridol $(1 \mu \mathrm{M})$ significantly counteracts the effects of TSA in terms of neurite length $(104 \mu \mathrm{m} \pm 16.8 \mathrm{~S} . \mathrm{D}$. TSA versus $65 \mu \mathrm{m} \pm 10.2 \mathrm{Flavopiridol}+\mathrm{TSA})$ and numbers (111 \pm 6.5 TSA versus $81 \pm 7$ Flavopiridol + TSA). Asterisks: unpaired two-tailed $t$-test, ${ }^{*} P$-value $<0.05$. Error bars represent S.D. (b) F-actin and $\beta$-III tubulin stainings allow the visualization of growth cones. Scale bar: $5 \mu \mathrm{m}$. In the growth cone collapse assay, non-collapsed growth cones were counted and represented by the bar graph as a percentage from three independent experiments ( $n$ : 100 cells each). The surface area of growth cones was also measured and quantified (three independent measurements, $n$ : 100 growth cones each). TSA increases the percentage of non-collapsed growth cones (18\% \pm 1.52 TSA versus $10 \% \pm 4.2$ vehicle) and the surface area $\left(113 \mu \mathrm{m}^{2} \pm 17\right.$ TSA versus $67 \mu \mathrm{m}^{2} \pm 11$ vehicle), whereas Flavopiridol promotes growth cone collapse with or without TSA in terms of percentage (18\% \pm 1.52 TSA versus $6 \% \pm 4.2$ Flavopiridol + TSA) and of surface area $\left(113 \mu \mathrm{m}^{2} \pm 17\right.$ TSA versus $21 \mu \mathrm{m}^{2} \pm 19$ Flavopiridol + TSA $)$. Asterisks: unpaired two-tailed $t$-test, ${ }^{\star} P$-value $<0.05$, ${ }^{* *} P$-value $<0.01$. Error bars represent S.D. (c) Immunoblotting analysis for GAP-43 shows an increase in GAP-43 protein expression after $24 \mathrm{~h}$ treatment with TSA (1 $\left.\mathrm{ng} / \mathrm{ml}\right)$, which is counteracted by Flavopiridol in addition to TSA. Flavopiridol itself reduces levels of GAP-43 protein expression. $\beta$-actin was used as a loading control

Actinomycin $\mathrm{D}$ and Flavopiridol were able to block the pro-outgrowth effects of TSA, showing that rescue of neurite outgrowth is transcription dependent (Supplementary Figure 3B).

Interestingly, when we measured global changes in $\mathrm{H} 3$ K9-14 acetylation in neurons plated on myelin versus neurons plated on PDL, we found no difference in H3 K9-14 acetylation (Supplementary Figure $3 \mathrm{C}$ ). This suggests that TSA may affect outgrowth by enhancing transcription via H3 K9-14 acetylation of specific promoters and not aspecifically throughout the genome.

Together, these data indicate that TSA significantly overcomes an inhibitory environment by promoting neuronal outgrowth, and by limiting growth cone collapse. This suggests that hyperacetylation might promote neuronal outgrowth on both permissive and inhibitory substrates in a 'substrate-independent' manner, perhaps by affecting the intrinsic transcription-dependent pro-growth program.

CBP, p300 and P/CAF are required for hyperacetylationdependent neuronal outgrowth. $\mathrm{H} 3 \mathrm{~K} 9-14$ acetylation is mediated by the HATs CBP/p300 and $\mathrm{P} / \mathrm{CAF},{ }^{23}$ which are also essential transcriptional co-factors involved in the overall protection of neurons from cell death in diverse stress conditions. ${ }^{7,10}$ 

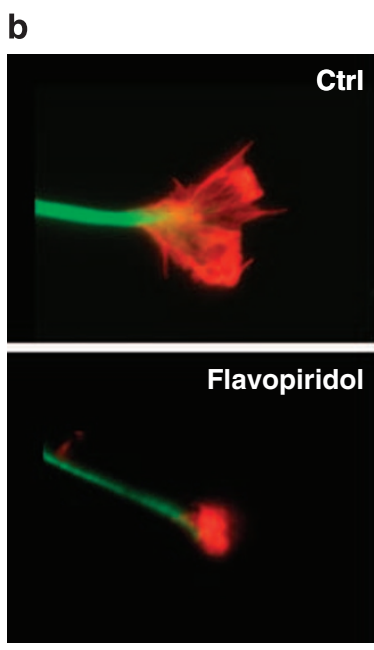

$\beta$ III Tubulin
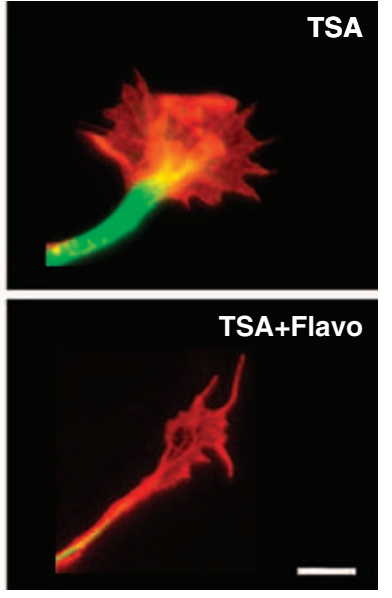

F actin
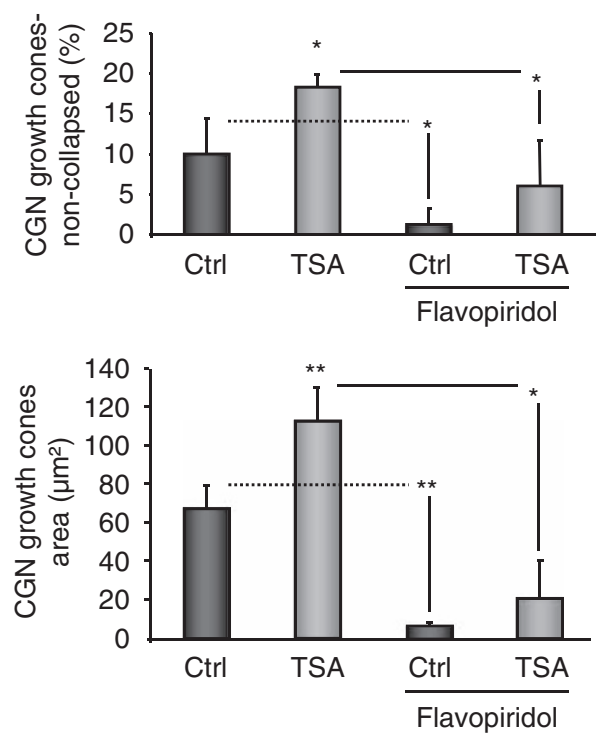

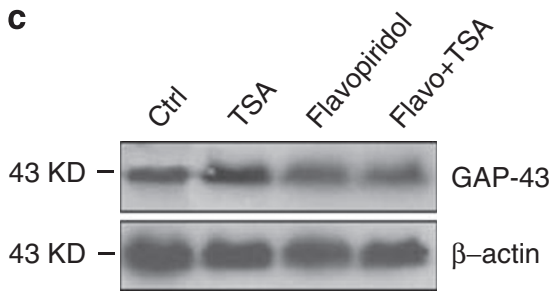

Figure 2 Continued

To investigate whether $\mathrm{CBP} / \mathrm{p} 300$ and $\mathrm{P} / \mathrm{CAF}$ are required for neuronal outgrowth, we have measured neuronal outgrowth in CGN after electroporation with specific RNA interference constructs and oligonucleotides to silence the expression of $\mathrm{CBP} / \mathrm{p} 300$ and $\mathrm{P} / \mathrm{CAF}$ after the administration of TSA or vehicle. Control for RNA interference was performed by transfecting scrambled sequences and naked DNA. Electroporated neurons were identified by co-transfection with GFP, and neuronal outgrowth was visualized by using antibodies against $\beta$-III-tubulin as a neuronal marker. Also, only CGN with strongly impaired expression by immunofluorescence for each of these HATs were included in the analysis. Gene silencing with these RNAi was robust as shown by RT-PCR and by immunofluorescence in individually electroporated CGN both in vehicle and TSA-treated cells (Supplementary Figure 4A-C). Importantly, CBP/p300 or $\mathrm{P} / \mathrm{CAF}$ gene silencing also correlated with reduced histone H3 K9-14 acetylation (Supplementary Figure 4D and E). In addition, cell viability was not affected by gene silencing as shown by counting the number of electroporated CGN with apoptotic features as shown by nuclear morphology and by the expression of cleaved-caspase 3 in control versus experimental conditions (Supplementary Figure 4F and $\mathrm{G}$ ).

Results showed that gene silencing of CBP/p300 and $\mathrm{P} / \mathrm{CAF}$ induces a significant decrease in TSA-mediated neuronal outgrowth, and in the average number of neurites/ 100 cells (Figure 4a). In addition, we observed that silencing of $\mathrm{CBP} / \mathrm{p} 300$ or P/CAF shows a tendency, but not a significant decrease in neurite length without TSA, as opposed to a significant decrease in the average number of neurites/100 cells.

Given that silencing of $\mathrm{CBP} / \mathrm{p} 300$ and $\mathrm{P} / \mathrm{CAF}$ on a permissive substrate inhibited the ability of TSA to induce neuronal outgrowth, we further investigated whether $\mathrm{CBP} / \mathrm{p} 300$ and P/CAF would also have a function in TSA-dependent outgrowth in neurons cultured on myelin, a potent inhibitor of neuronal outgrowth. We electroporated CGN with RNAi to silence CBP/p300 and P/CAF, and we detected neuronal outgrowth on myelin in analogous experiments to what we performed on permissive substrates. We found that silencing of both $\mathrm{CBP} / \mathrm{p} 300$ or P/CAF significantly reduced the 'recovery effects' of TSA and further enhanced the inhibitory properties of myelin as shown by total average neurite length in GFP positively transfected neurons; by the average number of neurites/100 cells; and by the percentage of non-collapsed growth cones (Figure 4b). Together, these data show that CBP/p300 and P/CAF are essential mediators of hyperacetylation-dependent neuronal outgrowth on both permissive and inhibitory substrates.

The evidence that gene silencing of $\mathrm{CBP} / \mathrm{p} 300$ or P/CAF abrogates the effect of TSA hyperacetylation in neuronal outgrowth, combined with the fact that hyperacetylation induces gene expression by facilitating transcription at specific promoters, led us to ask whether TSA is able to specifically promote gene expression of CBP/p300 and P/CAF. 
a

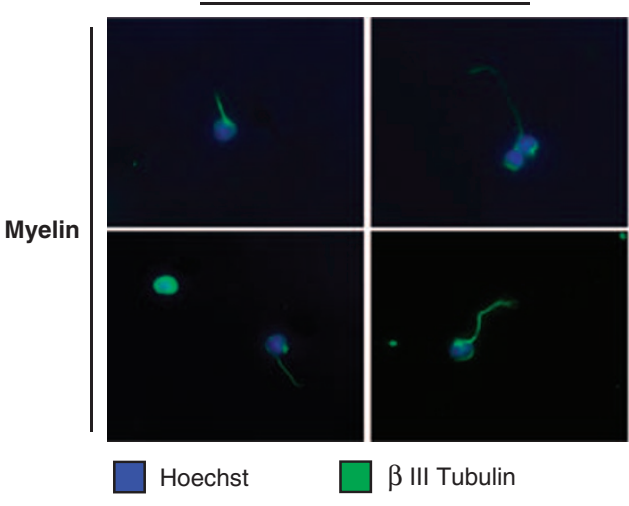

Ctr

b

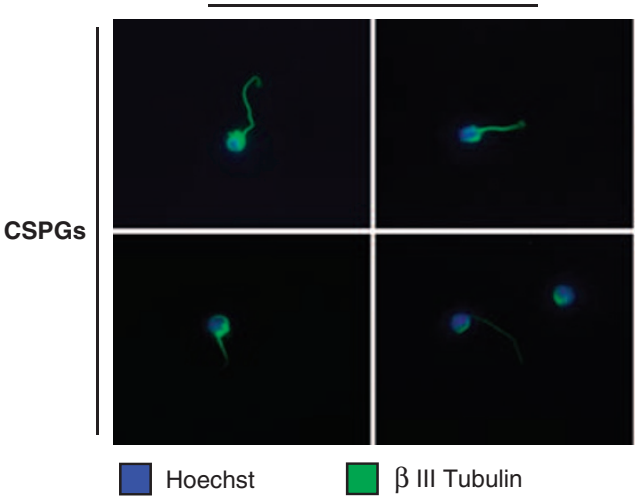

TSA

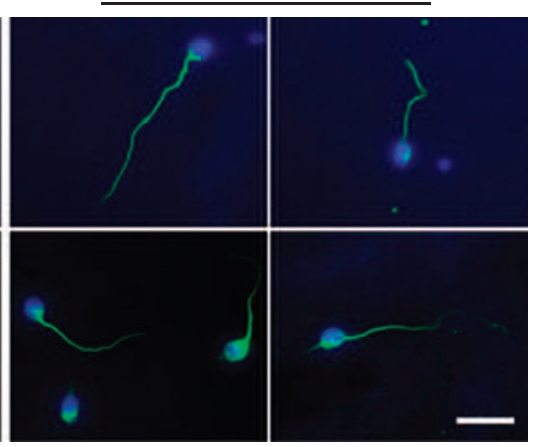

$-$

$\beta$ III Tubulin
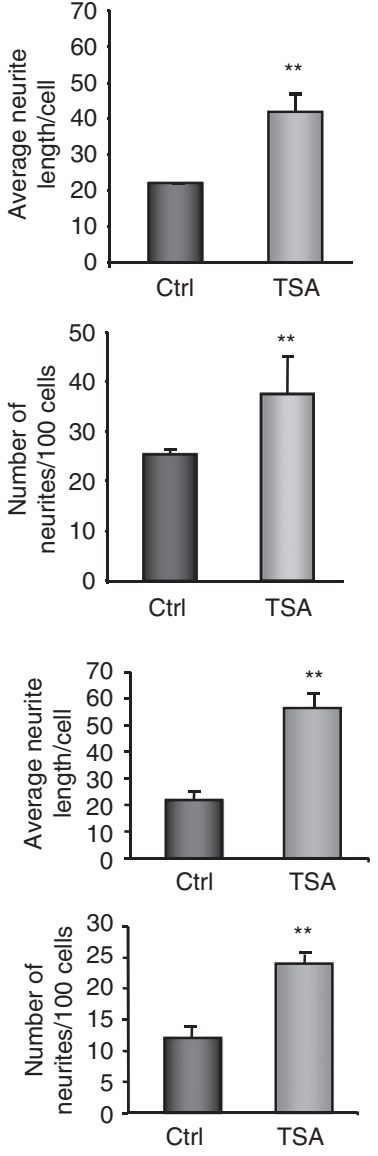
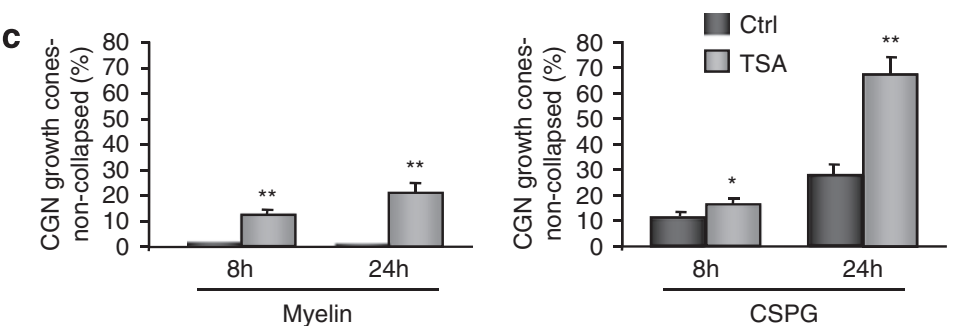

Figure 3 HDAC inhibition overcomes myelin and CSPGs inhibition by promoting neurite outgrowth and preventing growth cone collapse. (a, $\mathbf{b})$ Immunofluorescence of CGN double stained with $\beta$-III Tubulin and Hoechst shows the effects of $1 \mathrm{ng} / \mathrm{ml} \mathrm{TSA}$ treatment for $24 \mathrm{~h}$ on myelin (a) or CSPGs (b) versus vehicle (Ctrl). Scale bar: $20 \mu \mathrm{m}$. TSA treatment of CGN increases the average neurite length per cell ( $37.6 \mu \mathrm{m} \pm 7.5$ S.D. TSA versus $19.8 \mu \mathrm{m} \pm 1.6$ vehicle on myelin and $55 \mu \mathrm{m} \pm 5.1$ TSA versus $22 \mu \mathrm{m} \pm 3.2$ vehicle on CSPG) and the number of neurites per 100 cells (37.5 \pm 7.7 TSA versus $25 \pm 1.1$ vehicle on myelin and $24 \pm 1.4$ TSA versus $12 \pm 2$ vehicle on CSPG) as represented in the bar graphs of three independent measurements ( $n$ : 100 cells each). Asterisks: unpaired two-tailed $t$-test, ${ }^{\star \star} P$-value $<0.01$. Error bars represent S.D. (c) A growth cone collapse assay was performed on CGN plated either on myelin or CSPGs. The bar graphs represent the percentage of non-collapsed growth cones after 8 and $24 \mathrm{~h}$ of treatment on each substrate of three independent experiments ( $n: 100$ growth cones each). TSA increases the percentage of non-collapsed growth cones on both myelin and CSPG, respectively ( $24 \mathrm{~h}: 21 \% \pm 3.6$ TSA versus $1.5 \% \pm 1$ vehicle; $67 \% \pm 7$ TSA versus $27 \% \pm 4.8$ vehicle). Asterisks: unpaired two-tailed $t$-test, ${ }^{*} P$-value $<0.05$, ${ }^{*} P$-value $<0.01$. Error bars represent S.D.

Indeed, RT-PCR experiments showed that TSA induces a significant increase in gene expression for CBP, p300 and $\mathrm{P} / \mathrm{CAF}$ in cultured CGN (Figure 5a). Interestingly, we also found that gene expression of CBP, p300 and P/CAF was repressed by plating CGN on myelin as compared with $P D L$, and that TSA rescued this inhibition by triggering their overexpression (Figure 5b). As histone acetylation levels after TSA (see Figure 1c) are somewhat inhomogeneous showing significant difference from cell to cell, we investigated whether the acetylation levels of $\mathrm{H} 3 \mathrm{~K} 9-14$ correlated with the single cell expression of $\mathrm{CBP} / \mathrm{p} 300$ as well as with neurite outgrowth. Indeed, H3 K9-14 acetylation levels positively correlated with CBP and p300 expression and with the degree of neurite outgrowth (Supplementary Figure 5).

We then performed chromatin immunoprecipitation (ChIP) experiments in CGN to verify whether the gene expression changes of $\mathrm{CBP} / \mathrm{p} 300$ and $\mathrm{P} / \mathrm{CAF}$ correlated with an increase in the acetylation status of histone $\mathrm{H} 3$ on their promoter regions after TSA administration. ChIP followed by real-time quantitative PCR (qPCR) showed an increase of $\mathrm{H} 3$ 
a

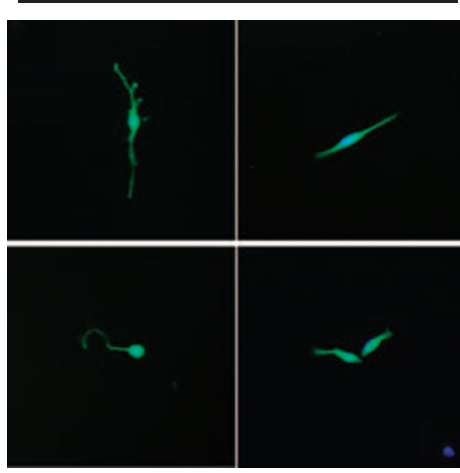

TSA
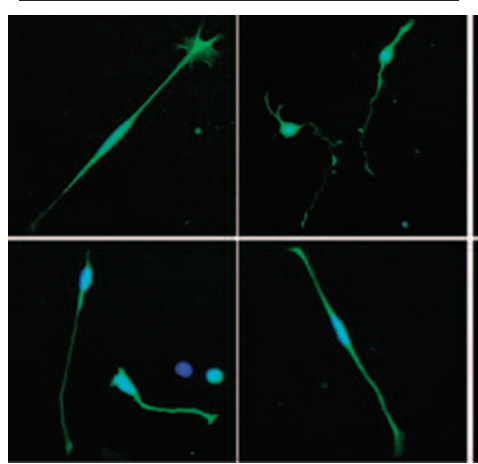

GFP

Hoechst
RNAi CBP/p300

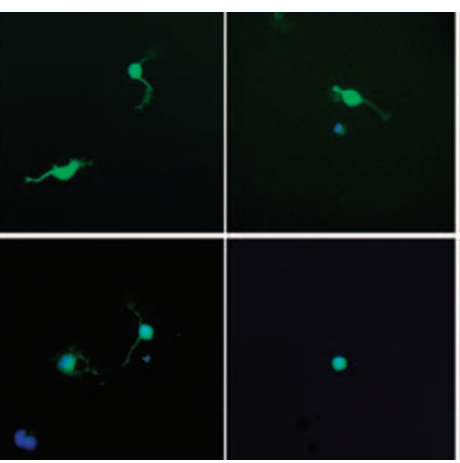

TSA+RNAi CBP/ p300

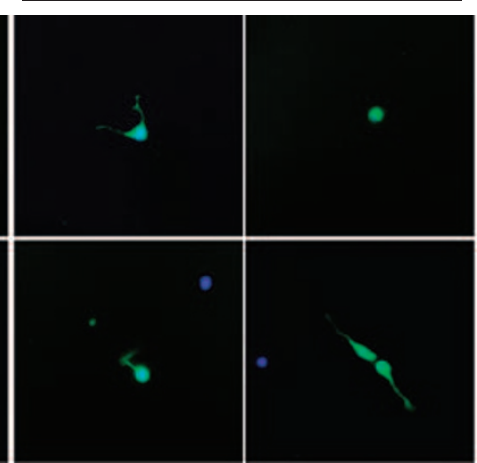

RNAi P/CAF

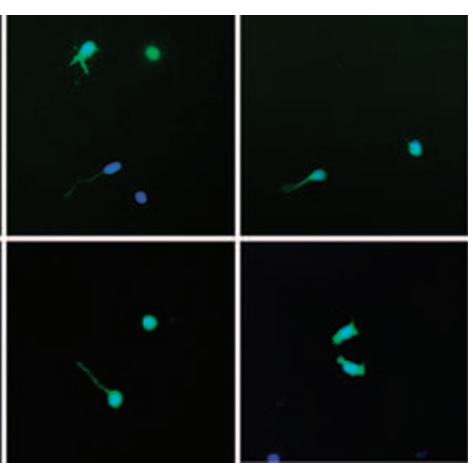

TSA+RNAi P/CAF

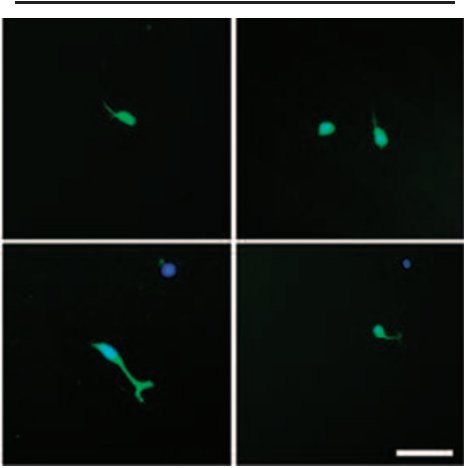

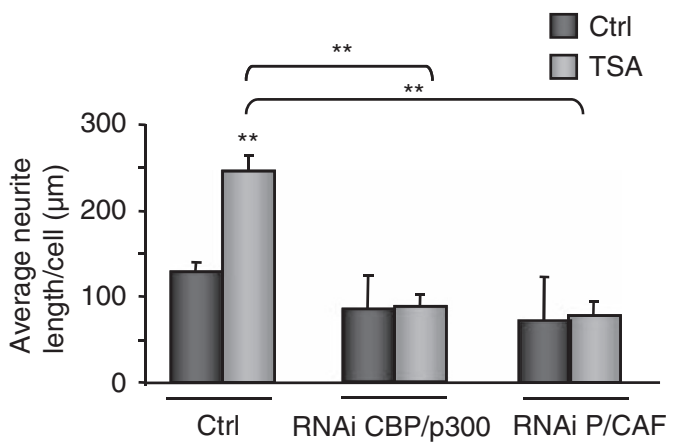

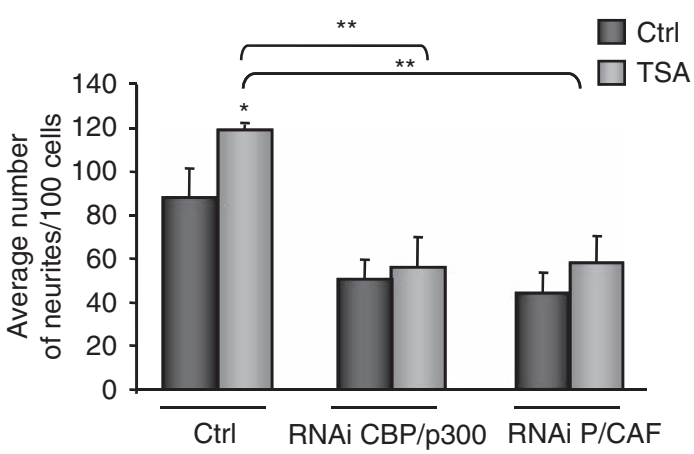

Figure $4 \mathrm{CBP} / \mathrm{p} 300$ and $\mathrm{P} / \mathrm{CAF}$ are required for hyperacetylation-dependent neurite outgrowth on permissive and non-permissive substrates. On a permissive substrate, such as PDL, immunofluorescence of CGN electroporated with either GFP-scrambled (ctrl) or GFP plus RNAi for CBP/p300 or shRNA for P/CAF in the absence or presence of $1 \mathrm{ng} / \mathrm{ml}$ TSA for $24 \mathrm{~h}$ show markedly reduced neurite outgrowth (total neurite length: $245 \mu \mathrm{m} \pm 18.4 \mathrm{~S}$.D. TSA/control versus $88 \mu \mathrm{m} \pm 15 \mathrm{TSA} / \mathrm{RNAi} \mathrm{CBP} / \mathrm{p} 300$, and $78 \mu \mathrm{m} \pm 16.5 \mathrm{TSA} / \mathrm{RNAi}$ P/CAF; average number of neurites/100 cells: $119 \pm 2 \mathrm{TSA} /$ control versus $56 \pm 14 \mathrm{TSA} / \mathrm{RNAi}$ CBP/p300, and $58 \pm 12$ TSA/RNAi P/CAF; $88 \pm 13$ control versus $51 \pm 8$ RNAi CBP/p300 and $44 \pm 9$ RNAi P/CAF) (a). Hoechst dye was used as a nuclear stain. The same result was observed when plated on the inhibitory substrate, myelin (total neurite length: $18.0 \mu \mathrm{m} \pm 2.5$ S.D. vehicle versus $57.4 \pm 2.6 \mathrm{TSA} ; 3.6 \pm 0.3$ vehicle $+\mathrm{RNAi} \mathrm{CBP} / \mathrm{p} 300$ versus $5.0 \pm 0.2 \mathrm{TSA}+\mathrm{RNAi} \mathrm{CBP} / \mathrm{p} 300$; $5.0 \pm 0.2$ vehicle + RNAi P/CAF versus $5.7 \pm 0.5 \mathrm{TSA}+\mathrm{RNAi} \mathrm{P} / \mathrm{CAF}$; average number of neurites/100 cells: $25 \pm 1.1$ vehicle versus $37.5 \pm 7.7 \mathrm{TSA} ; 11.45 \pm 4.3$ vehicle + RNAi CBP/p300 versus $13.7 \pm 3.5$ TSA + RNAi CBP/p300; $8.1 \pm 2.7$ vehicle + RNAi P/CAF versus $14 \pm 2.8$ TSA + RNAi P/CAF) (b). Co-immunolabelling for $\beta$-III tubulin was also used in these experiments (not shown). Scale bar: $30 \mu \mathrm{m}(\mathbf{a}) ; 15 \mu \mathrm{m}(\mathbf{b})$. Arrows in (b) show representative growth cones in control and TSA-treated neurons. Gene silencing of either $\mathrm{CBP} / \mathrm{p} 300$ or P/CAF significantly reduced average of neurite length per cell and number of neurites/100 cells on both PDL and myelin with and without TSA in three independent experiments ( $n$ : 100 cells each) as represented in the bar graphs $(\mathbf{a}, \mathbf{b})$. In addition, the percentage of non-collapsed growth cones from the neurons electroporated with either $\mathrm{CBP} / \mathrm{p} 300$ or P/CAF on myelin with or without TSA was significantly less than control GFP electroporated cells in three independent experiments ( $n: 100$ cells each) $(7.4 \% \pm 1.4$ vehicle versus $31.8 \pm 1.7 \mathrm{TSA} ; 1.6 \pm 0.4$ vehicle $+\mathrm{RNAi} C B P / p 300$ versus $4.7 \pm 2.1 \mathrm{TSA}+\mathrm{RNAi}$ CBP/p300; $1.1 \pm 0.3$ vehicle $+\mathrm{RNAi} / \mathrm{CAF}$ versus $4.3 \pm 0.5$ TSA + RNAi P/CAF) (b). Asterisks: unpaired two-tailed $t$-test, ${ }^{*} P$-value $<0.05$, ${ }^{* \star} P$-value $<0.01$. Error bars represent S.D.

acetylation at K9-14 on the promoters of CBP, p300 and P/CAF after stimulation with TSA compared with vehicle. This increase of $\mathrm{H} 3$ acetylation is not present on the promoter of the control gene RPL13a (Figure 5b). These data suggest that TSA induces gene expression of the HATs, CBP, p300 and P/CAF in CGN, by specific histone hyperacetylation on their promoters. 

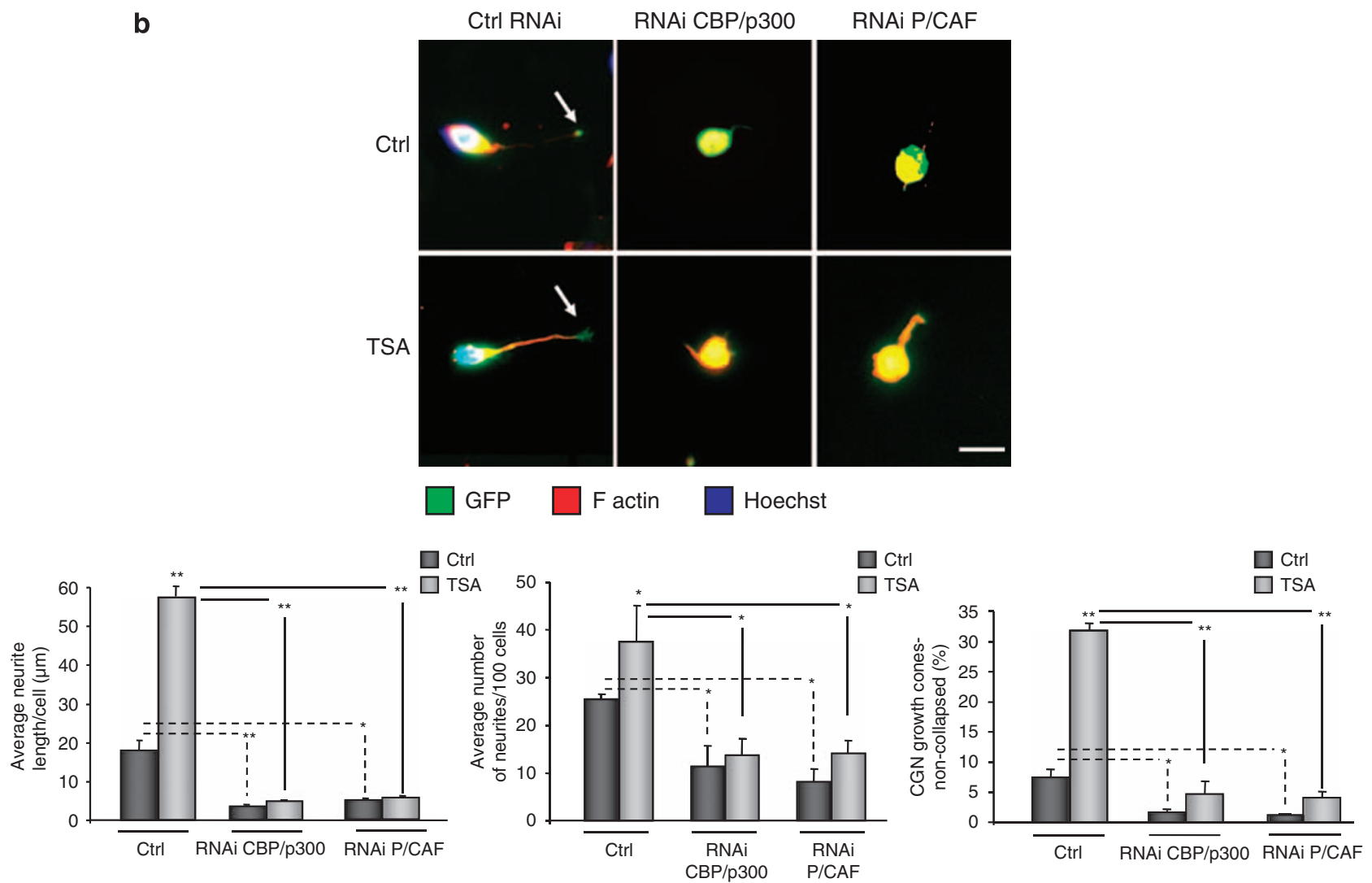

Figure 4 Continued

Hyperacetylation-mediated neuronal outgrowth is dependent on acetylated p53 signalling and drives pro-outgrowth gene targets. We have recently found that p53 forms pro-axonal outgrowth transcriptional complexes with $\mathrm{CBP} / \mathrm{p} 300$ and P/CAF on the promoter of pro-growth genes such as GAP-43 and Coronin 1b. ${ }^{18,19}$ We therefore examined whether p53 could transduce transcription signals within the pro-outgrowth hyperacetylation-dependent CBP/p300-P/CAF signalling cascade.

First, we examined whether p53 was acetylated after TSA treatment in CGN. Western blots of cultured CGN treated with TSA showed that p53 was indeed acetylated at K373 and $\mathrm{K} 320$, with peak in acetylation, respectively, at 8 and $4 \mathrm{~h}$ post-TSA (Figure 6a). Next, we measured the expression of the two previously described p53 transcription targets and pro-axonal outgrowth proteins GAP-43 and Coronin $1 \mathrm{~b}$ to assess whether in hyperacetylated neurons, p53-dependent pro-outgrowth signalling is activated. qRT-PCR and immunoblotting revealed that the gene and protein expression of Coronin $1 \mathrm{~b}$ and GAP-43 were induced after TSA treatment in hyperacetylated CGN (Figure $6 \mathrm{~b}$ and $\mathrm{c}$ ).

Then, ChIP assays were carried out in CGN to investigate the acetylation status of the promoters of these pro-axonal outgrowth factors by immunoprecipitation of DNA-protein complexes with an anti-acetyl H3 K9-14 antibody. Results showed that at both 8 (peak increase) and $24 \mathrm{~h}$, histones were acetylated on the promoters ( $5^{\prime}$ site) of GAP-43 and Coronin 1b. However, TSA did not induce further histone hyper- acetylation on these promoters at neither 8 nor $24 \mathrm{~h}$ (Figure 6d). Therefore, we hypothesized that the TSA-dependent acetylation of p53 would be responsible for the transcriptional activation of GAP-43 and Coronin 1b. To this end, we performed ChIP assays on CGN by using antibodies that recognize specifically acetylated p53 at lysine 320 and 373 and measured by qPCR the promoter occupancy of p53 on the promoters of Coronin $1 \mathrm{~b}$ and GAP-43 (respectively). Indeed, hyperacetylation increased the occupancy of p53 AcK373 on the promoters of both GAP-43 and Coronin 1b, whereas p53 AcK320 showed increased occupancy for Coronin 1b only. No occupancy was found on GAP-43 (Figure 6e). Together, these experiments show that after TSA treatment, p53 is hyperacetylated and the expression of specific p53-dependent prooutgrowth targets such as GAP-43 and Coronin $1 \mathrm{~b}$ are induced by increased promoter occupancy by acetylated p53 and not by histone hyperacetylation on their promoters, where histones are already acetylated in physiological growth conditions (Figure 6d).

To address the effects of hyperacetylation on driving the expression of several other well-known pro-neurite and axonal outgrowth genes that contain putative transcriptional binding sites for p53, we performed qRT-PCR experiments on selected targets, including SCG-10, $\alpha$-tubulin 1a, Chl1, L1cam, CAP-23 and Lgals1 (Supplementary Figure 6A and $B)$.

In fact, the expression of most of these putative p53 target genes, with the exception of CAP-23, was also driven in a 

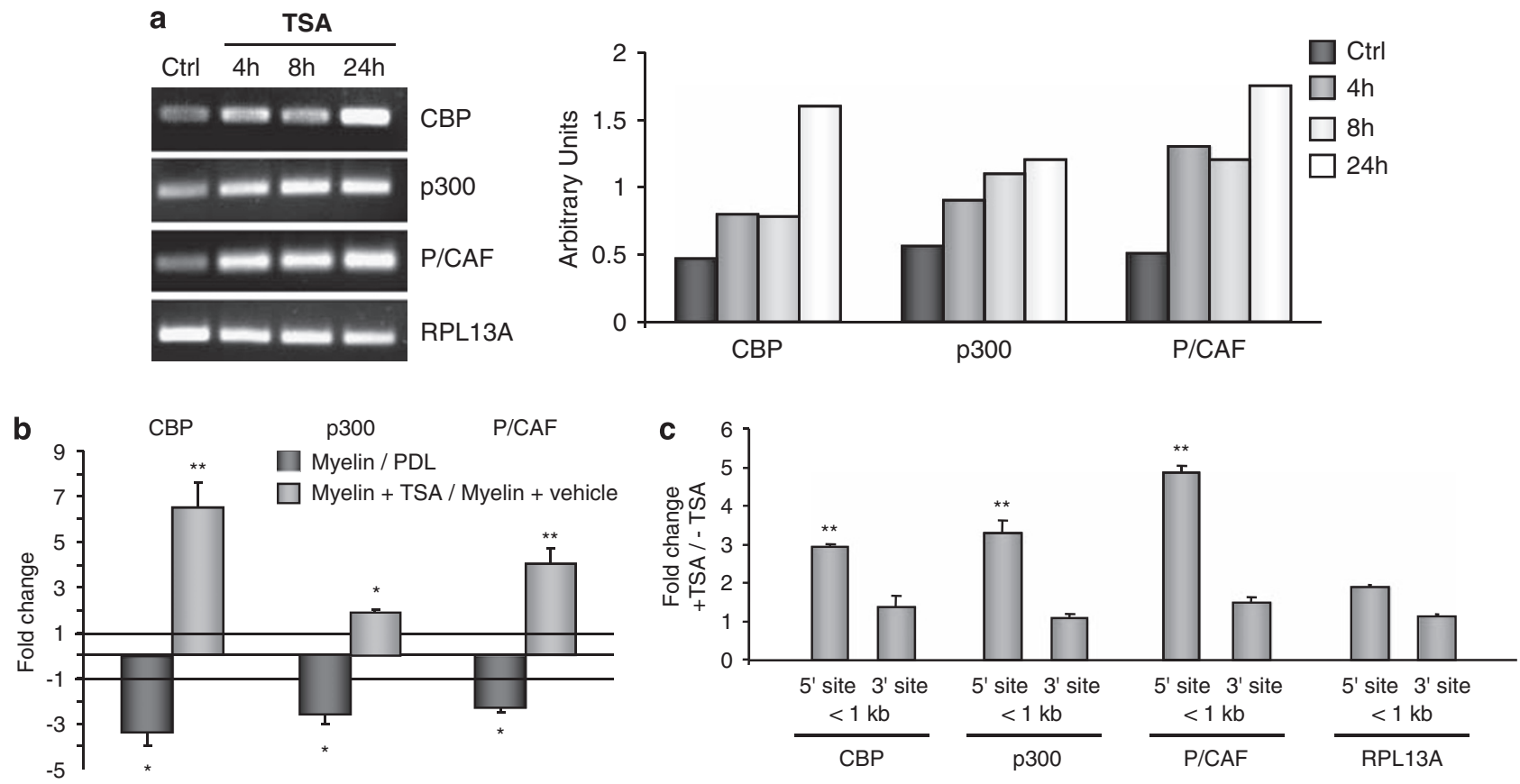

Figure 5 TSA-dependent hyperacetylation enhances the mRNA expression of $\mathrm{CBP} / \mathrm{p} 300$ and $\mathrm{P} / \mathrm{CAF}$ and promotes binding of acetylated $\mathrm{H} 3 \mathrm{~K} 9-14$ to their promoters. (a) Representative RT-PCR of mRNA levels for CBP, p300 and P/CAF. The upregulation of CBP, p300 and P/CAF mRNA observed by RT-PCR after $1 \mathrm{ng} / \mathrm{ml}$ TSA treatment versus vehicle is represented in the bar graph of densitometry (arbitrary units), normalized to the ribosomal RNA gene RPL13A. (b) Bar graph shows real-time RT-PCR gene expression data for CBP, p300 and P/CAF in CGN plated on myelin or PDL (24 h) and treated with vehicle or TSA (1ng/ml). Myelin represses gene expression of CBP, p300 and P/CAF (-3.3-fold \pm 0.5 S.D. CBP; -2.6 -fold \pm 0.4 S.D. $p 300$ and -2.3 -fold \pm 0.2 S.D. P/CAF compared to RPL13A), whereas TSA rescues myelin inhibition by inducing their overexpression (6.5-fold \pm 1 S.D. CBP; $1.9 \pm 0.1$ S.D. p300 and 4.02-fold \pm 0.6 S.D. P/CAF). Expression changes are quantified in fold changes as an average from three independent experiments. Asterisks: unpaired two-tailed $t$-test, ${ }^{*} P$-value $<0.05,{ }^{\star *} P$-value $<0.01$. Error bars represent S.D. (c) Chromatin IP (ChIP) assay in CGN shows increased occupancy of the CBP, p300 and P/CAF promoters by acetylated H3 K9-14 in comparison to the housekeeping gene RPL13A after $24 \mathrm{~h} 1 \mathrm{ng} / \mathrm{ml}$ TSA treatment versus vehicle (CBP: 2.95 -fold \pm 0.05 S.D., p300: 3.31 -fold \pm 0.2 and P/CAF: 4.9 -fold \pm 0.1 ). Fold change was calculated as a ratio of promoter occupancy between TSA-treated CGN versus vehicle in three independent experiments run in triplicate. The $3^{\prime}$ end regions of CBP, p300 and P/CAF were used as a control for specificity of acetylated $\mathrm{H} 3 \mathrm{~K}$ K-14 to the promoter regions. Asterisks: unpaired two-tailed $t$-test, ${ }^{\star \star} P$-value $<0.01$. Error bars represent S.D.

hyperacetylated environment (Supplementary Figure 6A and B), suggesting that multiple p53-dependent pro-neuronal outgrowth pathways could be activated by hyperacetylation.

Then, to evaluate whether acetylated p53-dependent cell signalling had a direct function in neuronal outgrowth during hyperacetylation, we electroporated CGN with a p53 mutant plasmid DNA, which carries a lysine to arginine transition at K320, K372-3-82 (p53 K320-72-3-82R, that we called here p53 K-R). These mutations do not allow acetylation of p53 by neither CBP/p300 (K372-3-82) nor by P/CAF (K320). The p53 $\mathrm{K}-\mathrm{R}$ transfected neurons did not display increased cell toxicity by cell counting nor they showed increased apoptotic features by Hoechst nuclear staining or cleaved caspase 3 expression compared with control transfected cells (Supplementary Figure 7A and B). Importantly, neurite measurements showed that neuronal outgrowth was significantly impaired in neurons transfected with $\mathrm{p} 53 \mathrm{~K}-\mathrm{R}$ both in the presence of TSA and in physiological culture conditions (Figure 7a). Electroporation of p53 K-R induced a significant decrease in the total average neurite length and an impaired number of neurites/100 cells (Figure 7a).

To further investigate whether the C-terminal acetylation of p53 would be required for hyperacetylation-dependent partial recovery of outgrowth in neurons cultured on inhibitory substrates, similar electroporation experiments were then performed on CGN plated on myelin.

Results showed that neurons expressing p53 K-R show a very strong reduction in outgrowth even in the presence of TSA. Total average neurite outgrowth as well as the average number of neurites $/ 100$ cells were markedly impaired in neurons transfected with p53 K-R as compared with GFP transfected only (Figure 7b). Furthermore, the percentage of collapsed growth cones was increased in the p53 K-R expressing neurons (Figure 7b).

To finally conclusively prove that $\mathrm{CBP} / \mathrm{p} 300, \mathrm{P} / \mathrm{CAF}$ and acetylated p53 are truly important to drive neurite outgrowth in primary neurons, overexpression experiments were performed on both PDL and myelin. Overexpression was carried out in cultured CGN via electroporation and neurite outgrowth was measured at $24 \mathrm{~h}$. A significant difference in outgrowth was observed when $\mathrm{CBP} / \mathrm{p} 300, \mathrm{P} / \mathrm{CAF}$ or the acetylation mimic p53 K-Q (K320-72-3-82 Q-lysine to glutamine) were overexpressed on either PDL or myelin (Figure 8). This shows that both on permissive substrates such as PDL and on myelin, their overexpression promotes a significant enhancement in outgrowth to levels comparable to the effects of TSA.

In summary, we have shown here that $\mathrm{CBP} / \mathrm{p} 300$ as well as $\mathrm{P} / \mathrm{CAF}$ and their related acetylation-dependent p53 signalling 
a

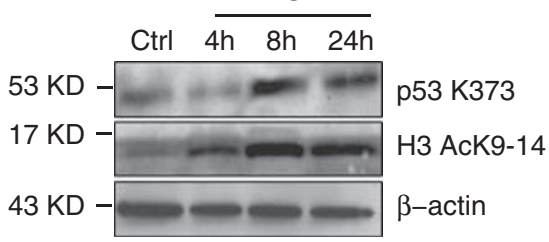

C

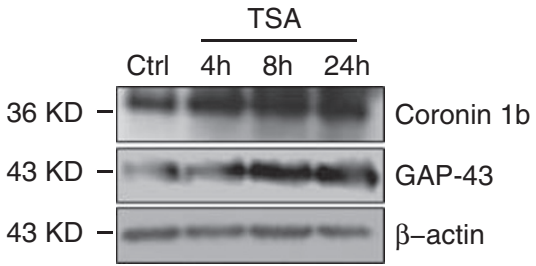

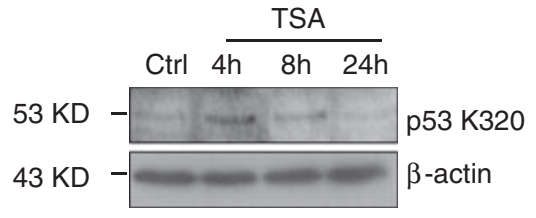

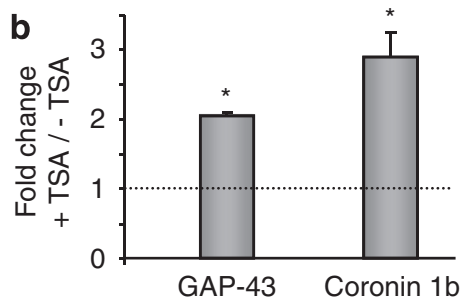

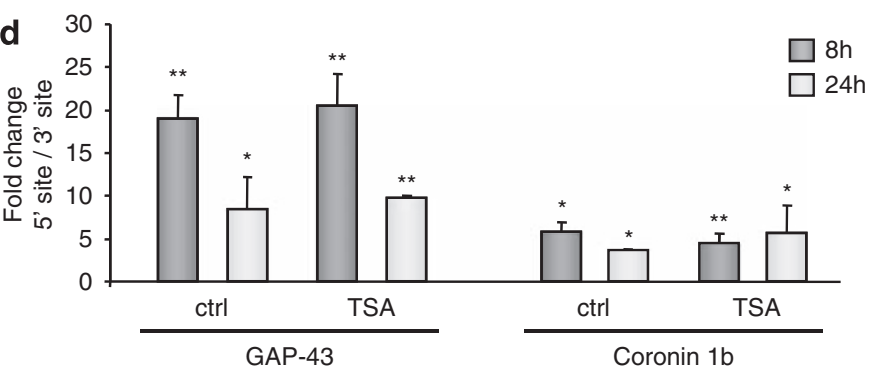

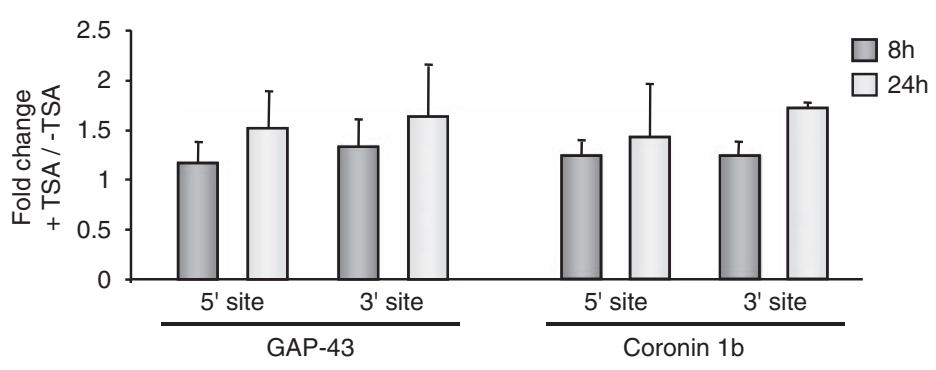

e

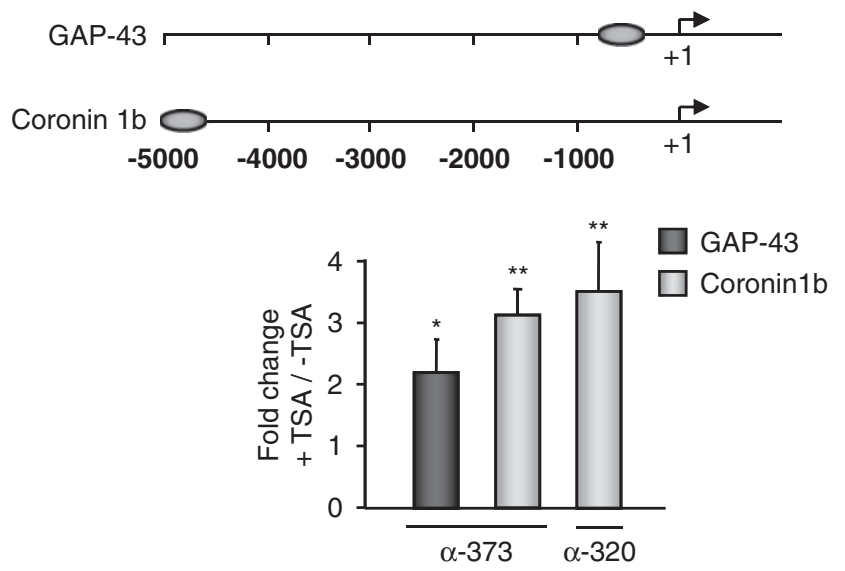

Figure 6 TSA hyperacetylates p53 and increases the expression of the p53 targets GAP-43 and Coronin 1b. (a) Immunoblotting analysis of CGN for acetylated p53 at K373 and K320 revealed that $1 \mathrm{ng} / \mathrm{ml}$ TSA induces p53 acetylation. (b) Real-time RT-PCR, as represented in the bar graph, shows a significant fold increase in GAP-43 and Coronin $1 \mathrm{~b}$ mRNA after $24 \mathrm{~h}$ treatment with $1 \mathrm{ng} / \mathrm{ml} \mathrm{TSA}$ versus vehicle in three independent experiments in triplicate (1.9-fold \pm 0.5 S.D. GAP-43 and 2.9-fold \pm 0.3 S.D. Coronin 1b). Asterisks: unpaired two-tailed $t$-test, ${ }^{*} P$-value $<0.05$. Error bars represent S.D. (c) Immunoblotting analysis shows an overexpression of both pro-axon outgrowth p53 target proteins GAP-43 and Coronin $1 \mathrm{~b}$ after treatment of CGN with $1 \mathrm{ng} / \mathrm{ml} \mathrm{TSA}$ versus vehicle (Ctrl). $\beta$-actin was used as a loading control. (d) Chromatin IP (ChIP) -realtime PCR assay (top panel) shows the presence of H3 K9-14 acetylation on the promoters of GAP-43 and Coronin $1 \mathrm{~b}$ in CGN at 8 and $24 \mathrm{~h}$ in culture (GAP-43 $8 \mathrm{~h}$ : 19 fold \pm 2.7 S.D. vehicle, $5^{\prime}$ versus $3^{\prime}$ site; 20 -fold \pm 4 TSA, $5^{\prime}$ versus $3^{\prime}$ site and Coronin $1 \mathrm{~b} 8 \mathrm{~h}$ : 5.7 -fold \pm 1.1 S.D. vehicle, $5^{\prime}$ versus $3^{\prime}$ site; 4.3 -fold \pm 1 S.D. TSA, $5^{\prime}$ versus $3^{\prime}$ site). However, in the same experiment, acetylation of their promoters is not enhanced by TSA (bottom panel). On the top panel, fold change was calculated as a ratio of promoter occupancy between the $5^{\prime}$ (promoter) versus $3^{\prime}$ site. On the bottom panel, fold change was calculated as a ratio of promoter occupancy between TSA versus vehicletreated CGN. This has been done in three independent experiments in triplicate. $3^{\prime}$ end regions of GAP-43 and Coronin $1 \mathrm{~b}$ were used as a control of the specificity of the acetylation of $\mathrm{H} 3$ at $\mathrm{K} 9$ - 14 on the promoter region. Asterisks: unpaired two-tailed $t$-test, ${ }^{*} P$-value $<0.05$, ${ }^{* \star} P$-value $<0.01$. Error bars represent $\mathrm{S}$.D. (e) A schematic diagram representing the position of the p53-binding sites on GAP-43 and Coronin $1 \mathrm{~b}$ (top panel). ChIP-real-time PCR assay (bottom panel) shows increased occupancy of acetylated p53 ( $\alpha$-320) on the promoter of Coronin $1 \mathrm{~b}(4 \mathrm{~h})(3.5$-fold \pm 0.8 S.D.), and acetylated p53 ( $\alpha-373)$ on the promoter of GAP-43 and Coronin $1 \mathrm{~b}$ (8 h) (2.2-fold \pm 0.5 S.D. GAP- 43 and 3.1 -fold \pm 0.4 S.D. Coronin $1 \mathrm{~b})$ in CGN after TSA $(1 \mathrm{ng} / \mathrm{ml})$. No promoter occupancy on GAP-43 was found for acetylated p53 $\alpha-320$. Fold change was calculated as a ratio of promoter occupancy between TSA-treated CGN versus vehicle in three independent experiments in triplicate. Asterisks: unpaired two-tailed $t$-test, ${ }^{*} P$-value $<0.05$, ${ }^{\star \star} P$ value $<0.01$. Error bars represent S.D. 

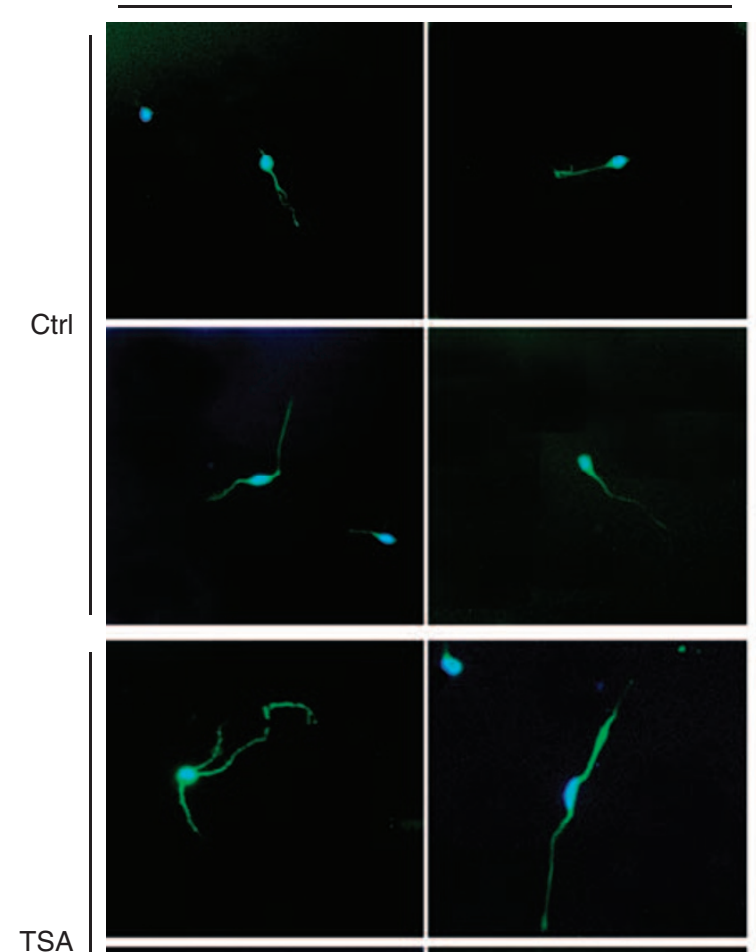

TSA

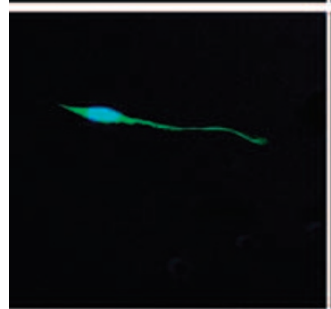

GFP $\square$ p53
Ctrl-GFP

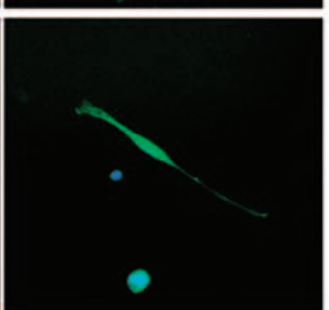

Hoechst
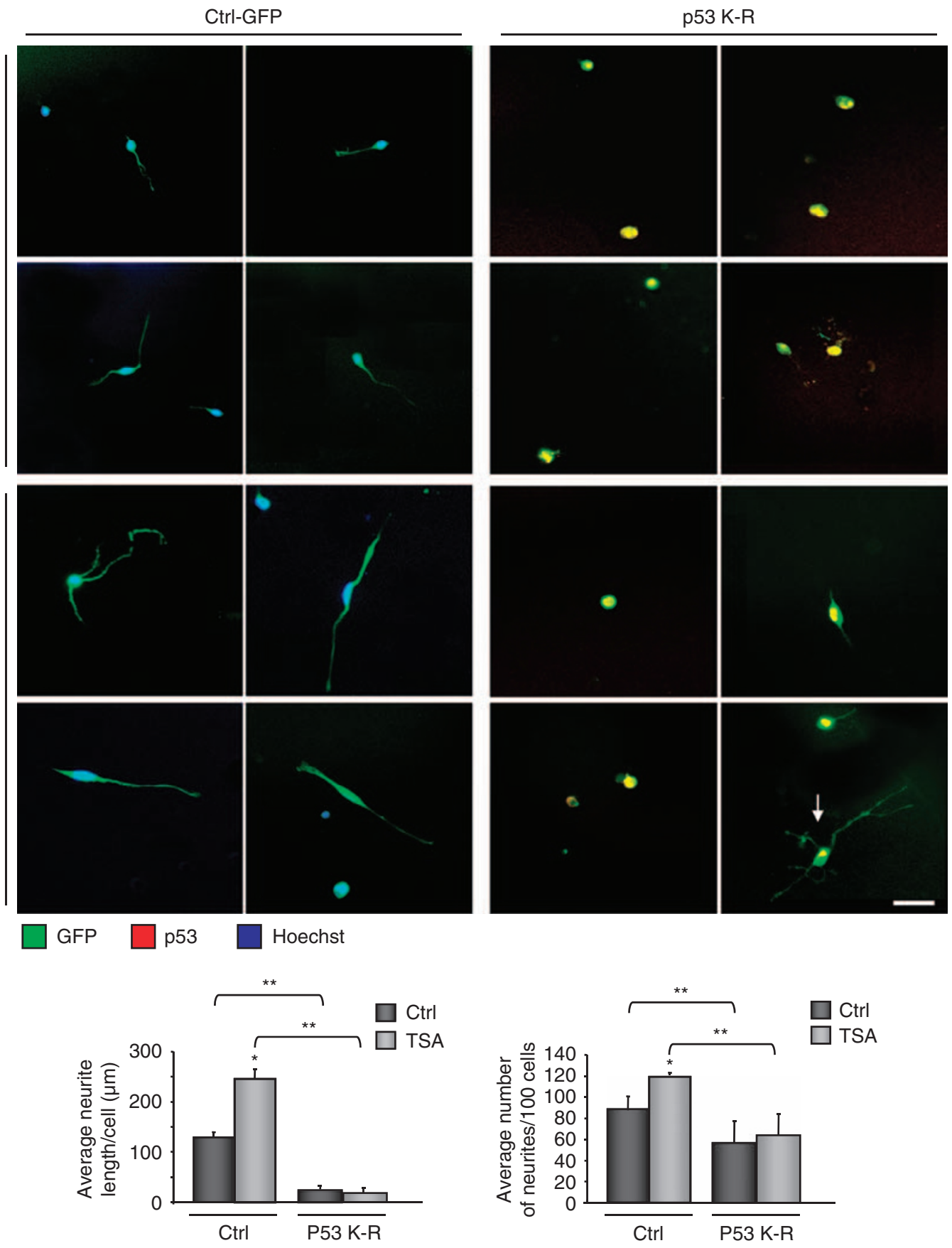

Figure 7 Hyperacetylation-mediated neurite outgrowth requires acetylation of p53 by CBP/p300 and PCAF. Immunofluorescence in CGN for p53 and Hoechst plated on PDL (a). CGN were electroporated with either GFP (ctrl) or GFP and a p53 mutant plasmid carrying K-R mutations at lysines 372-3-82 (p53 K-R) with or without $1 \mathrm{ng} / \mathrm{ml}$ TSA for $24 \mathrm{~h}$. In addition, these cells were plated on myelin (b) where they were stained with F-actin, for growth cone analysis, and Hoechst. $\beta$-III tubulin immunostaining was also used in these experiments (not shown). Neurons transfected with p53 K-R on both PDL (total average neurite length: $128 \mu \mathrm{m} \pm 10$ S.D. control versus $17 \pm 4.3$ p53 K-R; $245 \pm 18.5$ control/TSA versus $11 \pm 52$ p53 K-R/TSA; number of neurites/100 cells $88 \pm 13$ control versus $55 \pm 14$ p53 K-R; $119 \pm 2$ control/TSA versus $65 \pm 12$ p53 K-R/TSA) and myelin (total average neurite length: $5.17 \mu \mathrm{m} \pm 0.49$ S.D. p53 K-R/vehicle and $4.7 \pm 0.7$ p53 K-R/ TSA; average number of neurites $/ 100$ cells: $3.2 \pm 0.3$ S.D. p53 K-R/vehicle and $3.3 \pm 0.35$ p53 K-R/TSA) show impairment in neurite outgrowth and enhancement of growth cone collapse (only $1 \% \pm 0.57$ S.D. p53 K-R + vehicle and $1.33 \% \pm 0.75$ p53 K-R + TSA non-collapsed growth cones) (a, b). Importantly, when p53 K-R is expressed in the cytoplasm where it is transcriptionally inactive, physiological outgrowth does occur, neuron indicated by the arrow (a). Scale bar: $30 \mu \mathrm{m}(\mathbf{a}) ; 15 \mu \mathrm{m}$ (b). Arrows in (b) show representative growth cones in control and TSA-treated neurons. Measurement for average of neurite length per cell $(\mu \mathrm{m})$ and number of neurites/100 cells in three independent experiments ( $n$ : 100 cells each) are represented in the bar graphs $(\mathbf{a}, \mathbf{b})$. Asterisks: unpaired two-tailed $t$-test, ${ }^{*} P$-value $<0.05,{ }^{* *} P$-value $<0.01$. Error bars represent S.D. In addition, we quantified the percentage of non-collapsed growth cones in electroporated neurons plated on myelin, which showed a clear impairment in outgrowth in p53 K-R transfected cells with and without TSA in three independent experiments ( $n$ : 100 growth cones each). Asterisks: unpaired two-tailed $t$-test, ${ }^{\star *} P$-value $<0.01$. Error bars represent S.D. 
b
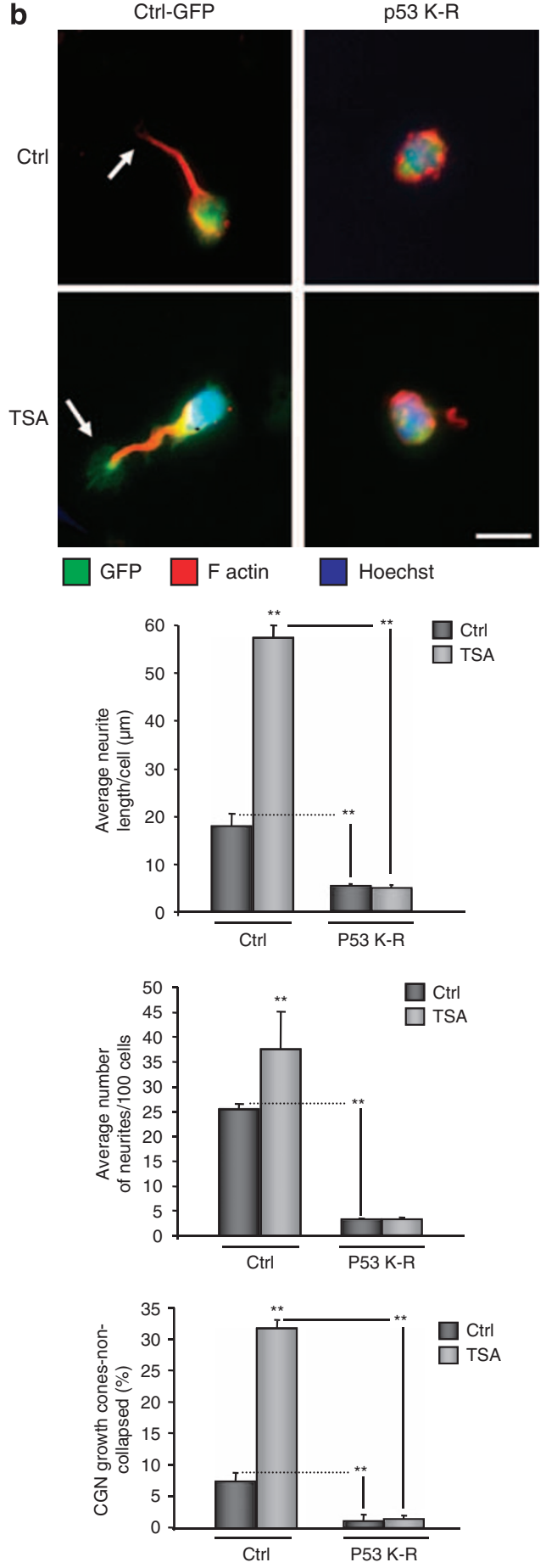

Figure 7 Continued

are key events in allowing physiological pro-outgrowth signals in primary neurons, and in driving outgrowth on inhibitory substrates, thereby transducing the hyperacetylation-dependent pro-outgrowth cascade on both permissive and non-permissive substrates (Figure 9, a diagram illustrating the molecular pathway elucidated here).

\section{Discussion}

Our work shows for the first time that pharmacological hyperacetylation mediated by HDAC I and II inhibition promotes neuronal outgrowth on both permissive and inhibitory substrates through induction of gene expression via histone $\mathrm{H} 3 \mathrm{~K} 9-14$ acetylation on the promoters of CBP/p300 and P/CAF, which in turn promote neuronal outgrowth and mediate $\mathrm{H} 3 \mathrm{~K} 9-14$ as well as p53 acetylation. These modifications lead to chromatin remodelling and enhanced p53-dependent promoter accessibility that drive the expression of specific pro-neuronal outgrowth genes.

Recent experiments in primary neurons showed that specific nitrosylation of HDAC2 increases acetylation of histones surrounding neurotrophin-dependent gene promoters and stimulate transcription. Inhibition of HDAC2 expression also facilitated dendritic growth and branching in embryonic cortical neurons. ${ }^{24}$ This supports our findings that inhibition of HDACs promotes neuronal outgrowth.

However, currently no studies have implicated CBP, p300 and the closely related P/CAF in neuronal outgrowth on permissive or inhibitory substrates. Here, we have shown that these HATs are required for physiological neuronal outgrowth and for the pro-outgrowth TSA-hyperacetylation-dependent signal transduction. Specifically, TSA promotes not only histone H3 K9-14 acetylation, but also stimulates CBP, p300 and $\mathrm{P} / \mathrm{CAF}$ gene expression by hyperacetylating their promoters, thereby facilitating transcription. This suggests the presence of a positive feedback loop initiated by the relative increase in acetyltransferase activity through inhibition of HDACs. This leads to histone hyperacetylation and activation of the CBP, p300 and P/CAF promoters, enhancement of gene expression further fueling this hyperacetylation loop. Given the shift in balance, the unchecked CBP, p300 and $\mathrm{P} / \mathrm{CAF}$ acetylate $\mathrm{p} 53$, which is essential to transduce the hyperacetylation-dependent pro-neuronal outgrowth program (Figure 9). In fact, the expression of specific p53-dependent genes, including GAP-43 and Coronin $1 \mathrm{~b}$, is triggered by hyperacetylation through increased occupancy of their promoters by acetylated p53. This may be facilitated by the presence of histone acetylation on their promoters that enhances the DNA accessibility of p53 and of its transcriptional co-factors.

Our data also suggest that hyperacetylation stimulates this pro-growth program independent of the plated substrate, for it also promotes growth cone remodelling and inhibits growth cone collapse in neurons when plated on inhibitory substrates (myelin and CSPGs). Importantly, overexpression of $\mathrm{CBP} / \mathrm{p} 300, \mathrm{P} / \mathrm{CAF}$ and of an acetylation mutant of p53 that mimics $\mathrm{CBP} / \mathrm{p} 300$ and $\mathrm{P} / \mathrm{CAF}$ acetylation on its $\mathrm{C}$-terminus promotes neurite outgrowth on permissive substrates and is able to overcome myelin inhibition. Acetylation of p53 mediated by CBP, p300 and P/CAF on its C-terminus redirects p53 towards specific promoters and cellular effects. ${ }^{25}$

Here, we show that the CBP/p300 and P/CAF-dependent acetylation of p53 is required for neuronal outgrowth and largely mediates the pro-outgrowth properties of TSA-induced hyperacetylation. Currently, we cannot exclude that other TFs that have been already shown to be acetylated and to be involved in neuronal survival or outgrowth, such as CREB and 
NFkB, ${ }^{15,17,26,27}$ might also participate in the TSA-mediated enhancement of neuronal outgrowth.

As far as the potential downstream targets are concerned, overexpression of GAP-43 and Coronin 1b might at least partially overcome myelin-dependent growth cone collapse by several potential mechanisms.

GAP-43 is a phospho-membrane bound glycoprotein, whose expression is often correlated with axonal outgrowth and regeneration in the peripheral nervous system, in the dorsal root ganglia, in the facial nerve nuclei, in the optic nerve and in the descending serotoninergic fibres in the spinal cord. It has also been shown to be associated with the overcoming of myelin and proteoglycan-dependent inhibitory signalling of axon outgrowth in the optic nerve after pro-regeneration stimuli. $^{28-31}$ In addition, cerebellar Purkinje cells that overexpress GAP-43 are able to sprout their axons into white matter and show a pronounced tendency to lose myelin. ${ }^{32}$ Coronin $1 \mathrm{~b}$ an actin-binding protein was previously shown by us to promote neurite outgrowth in primary neurons. ${ }^{33} \mathrm{~A}$ recent work suggested that Coronin $1 \mathrm{~b}$ can dephosphorylate cofilin during actin filament and cytoskeleton remodelling at the leading edge of lamellipodia. ${ }^{34}$ Phosphorylation of cofilin by ROCK and LIMK is a critical step in growth cone collapse mediated by inhibitory substrates, therefore the dephosphorylation activity of Coronin $1 \mathrm{~b}$ on cofilin may contribute to the inhibition of growth cone collapse. In addition to GAP-43 and Coronin $1 \mathrm{~b}$, the expression level of a set of putative p53-dependent pro-neurite outgrowth genes was also enhanced by TSA. This suggests the multiplicity of the p53 pathway during hyperacetylation on the activation of several pro-outgrowth factors.

Future challenges include the discovery of the fine regulation of the TSA-dependent effects on neuronal outgrowth. For example, it would be interesting to combine the administration of TSA with other compounds such as neurotrophins, including nerve growth factor, retinoic acid, brain-derived neurotrophic factor or CAMP analogues that might enhance TSA pro-neuronal outgrowth effects. Also, non-transcriptional-dependent effects of HDAC inhibitors could be investigated.

In summary, we believe that our work provides solid evidence for a positive function of HDAC inhibition and CBP/p300 and P/ CAF acetylation-dependent transcriptional pathways in neuronal outgrowth. Further studies are warranted to elucidate additional acetylation-dependent transcriptional targets that may promote outgrowth in vitro, and to investigate whether hyperacetylation may trigger a pro-axonal regenerative genetic program in adult neurons after axonal lesions in vivo.

\section{Materials and Methods \\ Drugs. TSA (T-8552, Sigma, Munich, Germany); PB (El-320, Biomol, lörrach, Germany), dbcAMP (CN-125, Enzo Life Sciences, lörrach, Germany), Actinomycin D (A1410, Sigma). Flavopiridol was provided by the Developmental Therapeutics Program, Division of Cancer Treatment and Diagnosis, National Cancer Institute, National Institutes of Heath.}

\section{Cell culture}

Primary cortical neurons. Rat cortical neuronal (RCN) cultures were derived from rat (CD) embryonic cortices. Cortices from 17-day-old embryos were extracted, dissociated and cultured as described earlier. ${ }^{35}$ Briefly, the cortices were minced and dissociated in a buffer containing $1800 \mathrm{U} / \mathrm{ml}$ trypsin at $37^{\circ} \mathrm{C}$ for $20 \mathrm{~min}$.
Followed by addition of $200 \mathrm{U} / \mathrm{ml}$ DNase I and $3600 \mathrm{U} / \mathrm{ml}$ soybean trypsin inhibitor to the suspension, cells were triturated through a 5-ml pipette. After the tissue was allowed to settle for 5-10 min, the supernatant was collected, and the remaining tissue pellet re-triturated. The combined supernatants were centrifuged through a $4 \%$ BSA layer, and the cell pellet was resuspended in neuronal seeding medium, which consisted of B27-Neurobasal Medium (Life Technologies, Darmstadt, Germany) supplemented with $1.1 \%$ of a $100 \times$ antibiotic-antimycotic solution, $25 \mu \mathrm{M}$ Na-glutamate, $0.5 \mathrm{mM}$ L-glutamine, and 2\% B27 Supplement (Life Technologies).

Primary CGNs. Cerebella granule neurons (CGN) were prepared from cerebella of 7-8-day-old rats (CD) as described earlier. ${ }^{36}$ Briefly, the cerebella were collected, and incubated for $15 \mathrm{~min}$ at $37^{\circ} \mathrm{C}$ in an ionic medium with $0.025 \%$ trypsin and $0.05 \%$ DNase I (Sigma). Then trypsin inhibitor $(0.04 \%$, Sigma) was added and the tissue was collected by centrifugation. The pellet was triturated, centrifuged and suspended in the growth medium, namely in the basal Eagle's medium supplemented with $10 \%$ bovine calf serum, $25 \mathrm{mM} \mathrm{KCl}, 4 \mathrm{mM}$ glutamine and gentamycin $(100 \mathrm{ng} / \mathrm{ml})$.

Immunocytochemistry. Glass coverslips were coated with PDL at $0.1 \mathrm{mg} / \mathrm{ml}$ for $2 \mathrm{~h}$ at $37^{\circ} \mathrm{C}$. For the experiment on myelin or CSPG, the glass coverslips were additionally coated with myelin $4 \mu \mathrm{g} / \mathrm{cm}^{2}$ and CSPG $1.25 \mu \mathrm{g} / \mathrm{cm}^{2}$ for $2 \mathrm{~h}$ at $37^{\circ} \mathrm{C}$. Cells were grown on glass coverslips for 8 and $24 \mathrm{~h}$. Cultured cells were fixed with $4 \%$ paraformaldehyde/ $4 \%$ sucrose. Cells were blocked in $10 \%$ albumin and $0.2 \%$ Triton-X100. Immunostaining was performed by incubation overnight at $4{ }^{\circ} \mathrm{C}$ with several antibodies: rabbit anti-acetylated-H3 (H3 AcK9-14, Upstate, Schwalbach, Germany); mouse anti- $\beta$-III Tubulin (Promega, Mannheim, Germany); rabbit anti-p53 (Ab1, Ab6, Oncogene, Darmstadt, Germany); mouse anti-MAP2 (Sigma); rabbit anti-TAU; rabbit anti-p300 (3G230); rabbit anti-CBP (AC238) (Abcam, Cambridge, UK); mouse anti-P/CAF (E-8) (Santa-Cruz, Heidelberg, Germany), rabbit anti-cleaved Caspase-3 (Cell Signalling, Frankfurt, Germany). Staining for F-actin was performed with the Alexa Fluor 568 Phalloidin (Molecular Probes, Karlsruhe, Germany) and incubated for $1 \mathrm{~h}$ at room temperature. After several rinses in PBS, the sections were incubated with the appropriate Alexa 488, 546 or 564-coupled secondary antibodies (goat anti-rabbit lgG, goat anti-mouse IgG, Pierce, Bonn, Germany) As a control, we stained with Hoechst 33258 (Molecular Probes) and then washed in PBS before mounting on slides with Fluorsave (Calbiochem, Darmstadt, Germany).

RT-PCR and qRT-PCR. RNA was extracted from CGN using TRIZOL reagent (Invitrogen) and CDNA was synthesized from $1 \mu \mathrm{g}$ of RNA using oligodT and random hexamers from the SuperScript II Reverse Transcriptase kit (Invitrogen). A measure of $1 \mu \mathrm{l}$ of CDNA was used in a RT-PCR using Master Mix (Invitrogen) and for qRT-PCR we used SYBR-greenER (Invitrogen).

The RPL13A gene was used for normalization. The sequences of the primers used were P/CAF forward $5^{\prime}$-TGGCGCACTTGTAGTACTCG- $3^{\prime}$ and reverse $5^{\prime}$ ATGGAACCAGTGAAGCGAAC-3' (124 bp), CBP forward 5'-ATCCCATAGACCCC AGTTCC-3' and reverse $5^{\prime}$-CGGCTGCTGATCTGTTGTTA-3' (206 bp), p300 forward $5^{\prime}$-GGGACTAACCAATGGTGGTG-3' and reverse $5^{\prime}$-ATTGGGAGAAGTC AAGCCTG-3' (386 bp), GAP-43 forward 5'-AAGCTACCACTGATAACTCGCC-3' and reverse $5^{\prime}$-CTTCTTTACCCTCATCCTGTCG-3' (246 bp); Coronin1b forward $5^{\prime}$-GACCTGTGCCCACATAACGATCAGG5C- $3^{\prime}$ and reverse $5^{\prime}$-CACGATGCCGA CTCTCTTTGA-3'; $\alpha$-Tubulin 1a forward $5^{\prime}$-GCTTCTTGGTTTTCCACAGC-3' and reverse $5^{\prime}$-TGGAATTGTAGGGCTCAACC-3' (162 bp); SCG10 forward 5'-CCACC ATTGCCTAGTGACCT-3' and reverse $5^{\prime}$-GAAGCACACACTCCACGAGA-3' (202 bp); Chl1 forward 5'-CGCCTACACAGGAGCTAAGG-3' and reverse 5'-TTCTITTGGAAGGCAGTGCT-3' (231 bp); L1cam forward 5'-CATCGCCTTTGTC AGTGCTA-3' and reverse $5^{\prime}$-CTGTACTCGCCGAAGGTCTC-3' (162 bp); Cap23 forward $5^{\prime}$-GGCGGCAGCGCTCCAACTCG- 3 ' and reverse $5^{\prime}$-CCGCCTGGGGTTC GCTCTCC-3' (304 bp); Lgals1 forward 5'-GCTGGTGGAGCAGGTCTCAGGAAT CT- $3^{\prime}$ and reverse $5^{\prime}$-AAGGTGATGCACTCCTCTGTGATGCTC- $3^{\prime}$ (314 bp); RPL13A forward $5^{\prime}$-CCCTCCACCCTATGACAAGA- $3^{\prime}$ and reverse $5^{\prime}$-CCTTTTCC TTCCGTTTCTCC-3' (167 bp). All primers were initially tested for their specificity by running RT-PCR samples on an agarose gel. Only primers that under specific PCR conditions gave a single band of the appropriate molecular weight were then used for real-time PCR experiments. Quantification of RT-PCR blot was performed by densitometry of the representative bands and normalized to the levels of the housekeeping gene (RPL13A) for each lane. For qRT-PCR, fold changes were calculated following the manufacture's instructions (Invitrogen, Karlsruhe, Germany). 
a

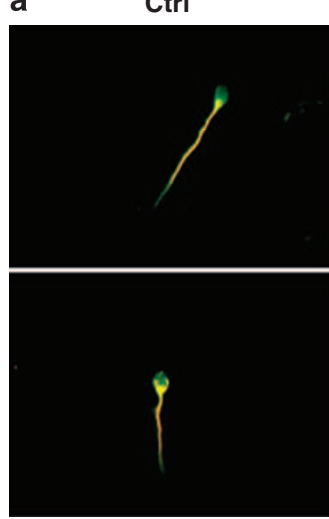

GFP
p53K-Q

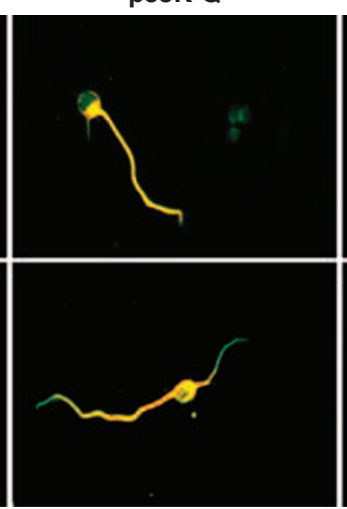

$\beta$ III tubulin

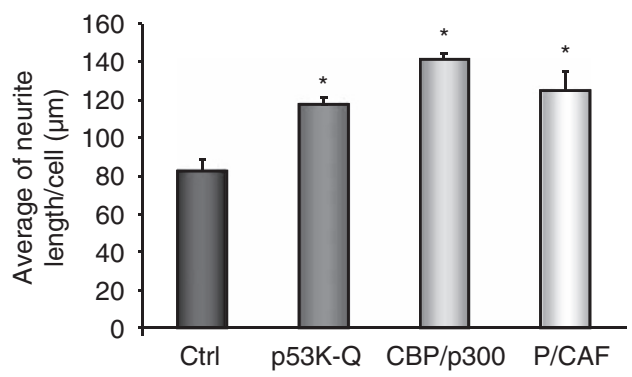

b

Ctrl

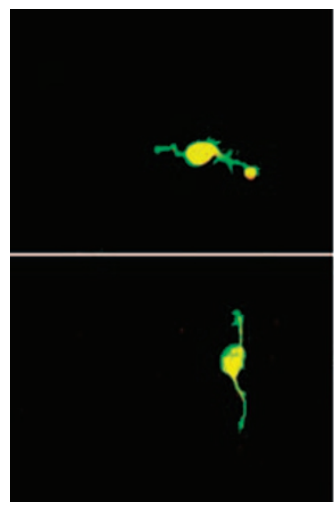

GFP

$\beta$ III tubulin
CBP/p300

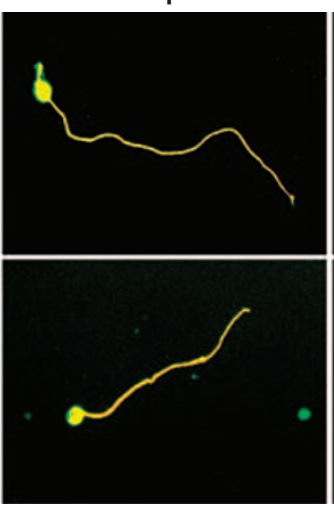

$-$

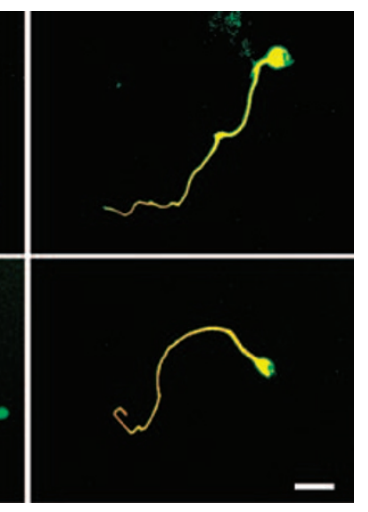

P/CAF

p53K-Q

CBP/p300

P/CAF
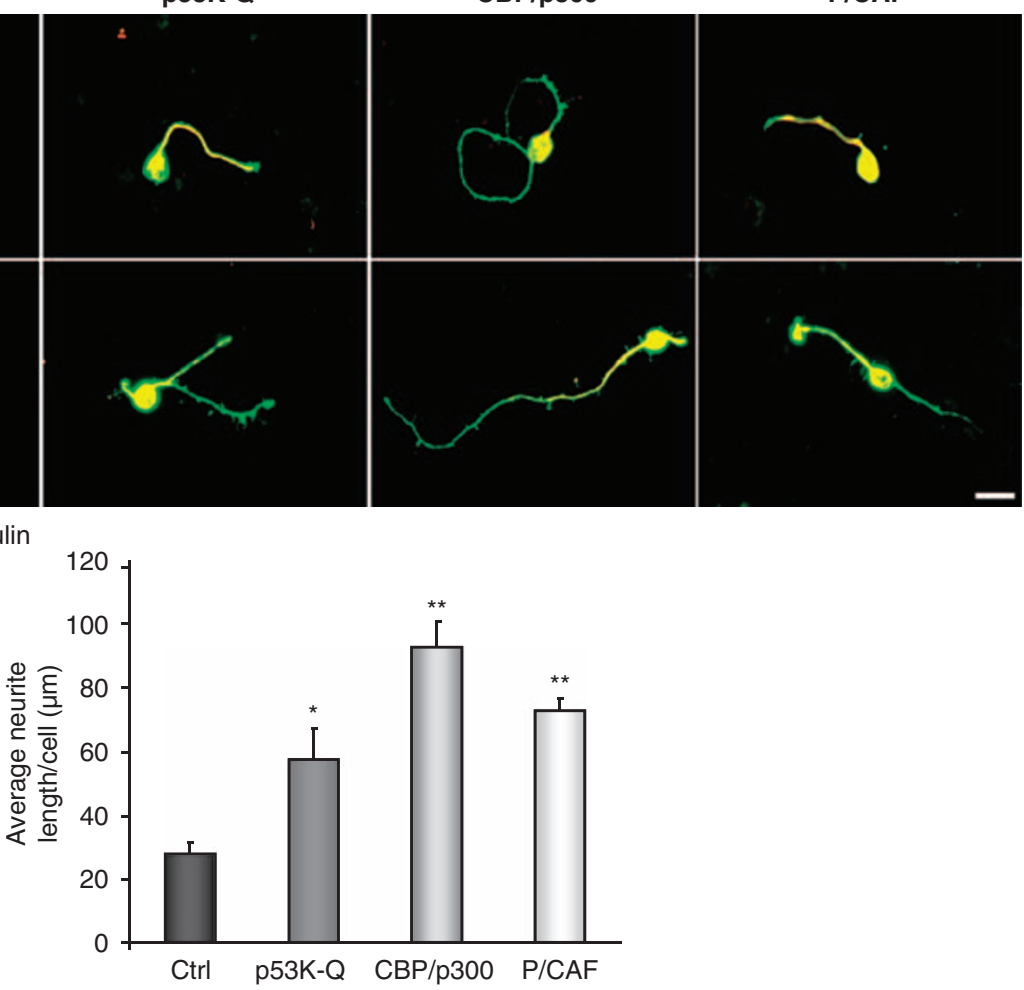

Figure 8 Overexpression of $\mathrm{CBP} / \mathrm{p} 300, \mathrm{P} / \mathrm{CAF}$ or $\mathrm{p} 53 \mathrm{~K}-\mathrm{Q}$ promote neurite outgrowth on permissive substrate and rescue the myelin-dependent inhibition of neurite outgrowth. (a) and (b) p53 K-Q (p53 K320-72-3-82Q), CBP-p300 and P/CAF were overexpressed in CGN plated on PDL (a) or myelin (b) for $24 \mathrm{~h}$. Cells were electroporated with either GFP and pcDNA 3.1 vector (ctrl); GFP and p53 K-Q; GFP and CBP/p300 plasmids, or GFP and P/CAF plasmid. The panels show immunocytochemistry for $\beta$-III tubulin and GFP signal. Scale bar: $20 \mu \mathrm{m}$. Bar graphs show quantification of neurite outgrowth, including average of length per cell (three independent experiments, $n: 100$ cells each). Neurons transfected with p53 K-Q, CBP/p300 or P/CAF show an increase in neurite outgrowth as compared with control (PDL: $117.4 \mu \mathrm{m} \pm 0.7 \mathrm{~S} . \mathrm{D}$. p53 KQ; $141 \mu \mathrm{m} \pm 3.2$ S.D. CBP/p300 and $124.7 \mu \mathrm{m} \pm 10.6$ P/CAF versus $82.2 \mu \mathrm{m} \pm 6.5$ S.D. pcDNA; Myelin: $57.4 \mu \mathrm{m} \pm 9.9$ S.D. p53 K-Q; $92.8 \mu \mathrm{m} \pm 7.9$ S.D. CBP/p300 and $73.0 \mu \mathrm{m} \pm 3.7$ S.D. P/CAF versus $27.9 \mu \mathrm{m} \pm 3$ S.D. pcDNA). Asterisks: unpaired two-tailed $t$-test, ${ }^{\star} P$-value $<0.05$, ${ }^{\star} P$-value $<0.01$. Error bars represent S.D. 


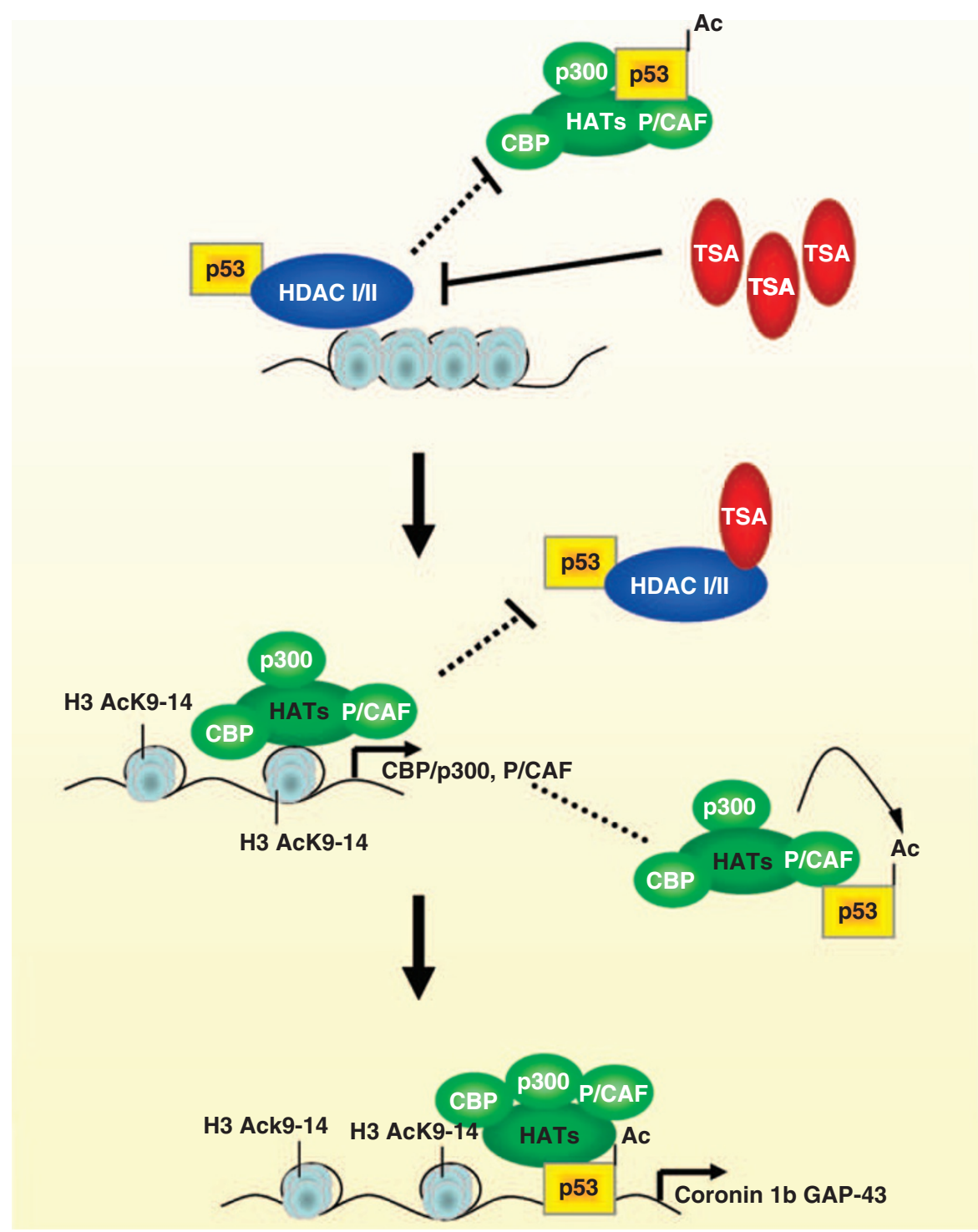

Figure 9 The hyperacetylation-dependent molecular pathway to neuronal outgrowth. In a non-permissive chromatin state, HDACs, class I and II, ensure that H3 remains hypoacetylated. Under these conditions, transcription complexes, including p53 and its corresponding cofactors, have a low affinity for promoters. When cells are treated with TSA HDACs are directly inhibited. This allows for the HATs, CBP/p300 and P/CAF, whose expression we found to be induced by TSA, to hyperacetylated H3 at K9-14 and p53 at its C-terminus, which then in concert with $\mathrm{CBP} / \mathrm{p} 300$ and $\mathrm{P} / \mathrm{CAF}$, occupy specific promoters within the newly formed permissive chromatin state. Finally, this promoter occupancy leads to the increased transcription of pro-growth genes involved in neuronal outgrowth

Gene silencing and overexpression experiments. The different $\mathrm{p} 53$, CBP, p300 and P/CAF expression constructs were transfected in CGN cells by using Amaxa electroporation kit, following the manufacturer's suggestions (Amaxa Nucleofector Technology, Cologne, Germany). For gene silencing, a maximum of $3 \mu \mathrm{g}$ of DNA was used, with a ratio of $0.5 \mu \mathrm{g}$ GFP/2.5 $\mu \mathrm{g}$ DNA and $10 \mathrm{nM}$ of siRNA for $\mathrm{CBP} / \mathrm{p} 300$. Gene silencing for P/CAF was performed by shRNA. For P/CAF gene silencing by shRNA, both hairpin sequences, which were designed and synthesized (Invitrogen) with specific overhangs for cloning into the pENTR/U6 entry vector, and oligonucleotides were used. The sequences of the 2 primer pairs used for P/CAF silencing were:

1: ORF1 5'-CACCGCTCGTTTGACCCGAAACACGAATGTTTCGGGTCAAA GAC GAGC-3', ORF2 5'-AAAAGCTCGTCTTTGACCCGAAACATTCGTGT TTCGGGTCAAAGACGAGC-3';

2: ORF1 5'-CACCGCCTCAGGAACAGGTACTATGCGAACATAGTACCTGTT CCTG AGGC-3'; ORF 2 5'-AAAAGCCTCAGGAACAGGTACTATGTTCGCA TAGTACCTGTTCCTGAGGA-3'.
siRNA oligonucleotides were used for rat CBP and p300 gene silencing and were a gift from Dr. Katsuhide Miyake. ${ }^{37}$

siRNAs with the following sense and antisense sequences were used:

CBP forward $5^{\prime}$-CCCACAGCUAAUGGCAGCUdTdT- $3^{\prime}$ and reverse $5^{\prime}$-AGCU GCCAUUAGCUGUGGGdTdT-3', p300 forward 5'-CCCCAUGGAACAGCAU dTdT-3' and reverse $5^{\prime}$-AUGCCCUUGGUUUUCCAUGGGGdTdT-3'.

The inhibitory effect of RNA interference on expression of CBP, p300 was tested by RT-PCR and in CGN by immunofluorescence that allowed quantification in individually electroporated cells.

The overexpression experiments were performed by electroporating CGN with pcDNA 3.1 vector as control, pCMVb p300 HA (Addgene, plasmid 10718), pRc/RSV CBP HA (Addgene, plasmid 16701) and pCl flag PCAF (Addgene, plasmid 8941) at the same ratio GFP/DNA as used for gene silencing experiments. p53 K320-72-3-82 R (p53 K-R) and p53 K320-72-3-82 Q (p53 K-Q) were cloned in a CMV promoter with a GFP tag and transfected by electroporation as described. 
ChIP assays. ChIP assays were performed according to the manufacturer's recommendations (Upstate). Briefly, $8 \times 10^{7} \mathrm{CGN}$ were grown in the absence or presence of TSA $(1 \mathrm{ng} / \mathrm{ml})$ for $24 \mathrm{~h}$ and subsequently fixed in a $1 \%$ formaldehyde solution for $10 \mathrm{~min}$ at $37^{\circ} \mathrm{C}$. Following cell lysis $(0.5 \% \mathrm{SDS}, 100 \mathrm{mM} \mathrm{NaCl}, 50 \mathrm{mM}$ Tris $\mathrm{HCl}$, ph 8.0, $5 \mathrm{mM}$ EDTA), extracts were sonicated to shear DNA to lengths of $200-600 \mathrm{bp}$.

Chromatin solutions were incubated overnight with rotation using $4 \mu \mathrm{g}$ of rabbit polyclonal anti-Acetyl histone H3 antibody (Upstate), anti-p53 acetyl-lysine K373/2 (Upstate) or rabbit anti-p53 acetyl-lysine K320 (Upstate). The following day protein A agarose beads, that had been blocked with salmon sperm DNA, were added to each reaction to precipitate antibody complexes. The precipitated complexes were washed and then incubated for $4 \mathrm{~h}$ at $65^{\circ} \mathrm{C}$ in parallel with input samples to reverse the crosslink. DNA was isolated by P:C:I extraction, which was followed by ethanol precipitation in the presence of sodium acetate.

Input, IP and Mock fractions were then analysed by PCR on an agarose gel to detect the presence of the single band of the appropriate molecular weight in Input and IP fractions, as well as its absence in Mock samples. This allows evaluating for both the specificity of the primers and of the immunoprecipitation. Only samples that respected these conditions were then analysed by quantitative real-time PCR (ABI 7000) analysis with appropriate primer pairs. The primers used were as follows: CBP $5^{\prime}$ site $<1 \mathrm{~kb}$ forward $5^{\prime}$-CACAGGTTTCTCGCTTAGGG-3' and reverse 5'-CCAAGGGCTTCGATGTTTTA-3'; CBP 3'site forward 5'-CTGGGGACATCCTTTT CAGA- $3^{\prime}$ and reverse $5^{\prime}$-CTGGGCACTCCAGCTCTTAC-3'; p300 $5^{\prime}$ site $<1 \mathrm{~kb}$ forward $5^{\prime}$-GCCCCTACCATTTCTGTTGA-3' and reverse $5^{\prime}$-CGGAGATACAACCG AGGAAA-3'; p300 3'site forward 5'-CCTGCACTTAGAGGCAAAGG-3' and reverse $5^{\prime}$-TGCTGCCTGAATGAGTTGTC-3'; PCAF $5^{\prime}$ site $<1 \mathrm{~kb}$ forward $5^{\prime}$-ATGTGTCCC CTGGCTTTATTCA-3' and reverse $5^{\prime}$-ACCCTCAGTTCGGTCTCCTT-3'; PCAF $3^{\prime}$ site forward $5^{\prime}$-CTGGCATGTCCAGTAGCTGA-3' and reverse $5^{\prime}$-TCACCGCACA GGTGTATCAT-3'; Coronin $1 \mathrm{~b} 5^{\prime}$ site $<1 \mathrm{~kb}$ forward $5^{\prime}$-CTCCCAGCGTTATCATG TCA-3' and reverse 5'-GGGAGACTCGAATGTCCTCA-3'; Coronin 1b $3^{\prime}$ site $\sim 10 \mathrm{~kb}$ forward $5^{\prime}$-ACTGTTGTGGAGGGCTATGG-3' and reverse $5^{\prime}$-TGGAGGTC AGATGCCTCTCT-3'; GAP-43 5' site < 1 kb forward 5'-GCAGCTGTAACTTGTGT GCA- $3^{\prime}$ and reverse $5^{\prime}$-GGTCCAGATTGGAGGTGTTTA-3'; GAP-43 $3^{\prime}$ site $\sim 10 \mathrm{~kb}$ forward $5^{\prime}$-TTCCTTAGGCAATGTTTTGGAAAG- $3^{\prime}$ and reverse $5^{\prime}$-TCAGGCATGT TCTTGGTCAG-3'; RPL13A $5^{\prime}$ site $<1 \mathrm{~kb}$ forward $5^{\prime}$-TTCAGCACACCAAGAGGT TG-3' and reverse $5^{\prime}$-GTTCAGGTGTCCGTGATGTG-3'; RPL13A $3^{\prime}$ site $\sim 10 \mathrm{~kb}$ forward $5^{\prime}$-GGCCATAAACATCTGGGAGA- $3^{\prime}$ and reverse $5^{\prime}$-GGGCAAGAGAGAC TCAGGTG- ${ }^{\prime}$. For real-time quantitation of PCR products and fold change measurements after ChIP, each experimental sample was normalized to Input and Mock fractions before undergoing the standardized fold change calculation performed following the manufacture's instructions (Invitrogen). Only real-time expression profiles that showed reproducible triplicate measurements were included in the analysis. In addition, samples included in the analysis had corresponding Mock fractions that displayed CT values close to background.

Immunoblotting. For immunoblotting, cultured CGN were collected at different time point (4, 8 and $24 \mathrm{~h})$ and lysed as described above for tissue extraction. A portion of the lysate (30-50 $\mu \mathrm{g}$ of protein) was then fractionated by SDS-polyacrylamide gel electrophoresis, and the separated proteins were transferred to a PVDF membrane. After the transfer of samples, the membrane was then probed with rabbit anti-acetylated-H3 (K9, K14, Upstate), rabbit anti-p53 acetyl-lysine K373/2 (Upstate), rabbit anti-p53 acetyl-lysine K320 (Upstate), rabbit anti-p53 FL (Santa-Cruz); rabbit anti-GAP-43 (Chemicon, schwalbach, Germany) and rabbit anti-Coronin $1 \mathrm{~b}$ (custom antibody from Bethyl Laboratories, Montgomery, TX, USA). Mouse anti- $\beta$-actin (Sigma) was used as control for gel loading and transfer. Immune complexes were detected with appropriate secondary antibodies (goat anti-rabbit $\lg G$, goat anti-mouse $\lg G$, label with horseradish peroxidase, Thermo Scientific, Bonn, Germany) and chemiluminescence reagents (Pierce ECL Western blotting Substrate). Quantitation of protein expression was performed by densitometry of the representative bands of the immunoblots and normalized to the respective levels of $\beta$-actin.

Adult primary neurons from the cortex or the cerebellum were also prepared as mentioned before for RCN or CGN cultures, respectively. After dissociation, cells were immediately collected by centrifugation and homogenized for immunoblotting as described. This procedure results into an enriched neuronal population at the time of blotting.

Neuronal process outgrowth measurements. Immunofluorescence was detected using a Zeiss Microscope (Axiovert 200, Zeiss Inc., Esslingen,
Germany) and pictures were taken at a $20 \times$ and $100 \times$ (oil immersion) magnification (Zeiss) at room temperature using a CDD camera (Axiocam MRm, Zeiss). Neurite analysis and measurements were performed using the Neurolucida software (MicroBrightField, Colchester, VT, USA). For each experimental condition and three replicates in triplicate experiments were analysed. Between 80 and 100 cells per replicate were counted (for specific numbers, please see figure legend). Neurite outgrowth was evaluated by calculating the number of processes per 100 cells and the average process length per cell. A growth cone was defined collapsed when the diameter was not bigger than the diameter of the neuronal process. The area of the non-collapsed growth cones was measured on an average of 100 growth cones for each triplicate. Measurements were taken from three individual experiments by two different operators. Statistical analysis was performed on these three individual experiments by using a two-tailed unpaired $t$-test.

Densitometry analysis. To correlate histone acetylation and neurite outgrowth, we used densitometric analysis of the immunofluorescent signal (AlphaEaseFC software). First, we calculated the mean intensity for AcH3 K9-14 in control (vehicle) and TSA (1 ng/ml)-treated CGN cells (50 cells per experiments were counted, in triplicate). Each mean intensity was then used as a threshold to determine cells with a high or low intensity of AcH3 K9-14 immunosignal after TSA. Cells with a signal equal or superior to $\mathrm{AcH} 3 \mathrm{Kg}-14$ mean intensity of TSA-treated cells were considered as high intensity cells. On the contrary, cells with a densitometry value equal or inferior to $\mathrm{AcH} 3 \mathrm{Kg}-14$ mean intensity of control were considered as low intensity cells. Cells showing pyknotic nuclei were not included in the study. Neurites outgrowth was then quantified in these two classes of cells and compared as described above. Statistical analysis was performed on these three individual experiments by using a two-tailed unpaired $t$-test.

\section{Conflict of interest}

The authors declare no conflict of interest.

Acknowledgements. We thank Dr. Maria Laura Avantaggiati for providing the p53 acetylation mutant plasmid DNAs, and Dr. Katsuhide Miyake for providing the CBP/p300 RNAi. We also thank Dr. Richard Goodman for providing the CBP plasmid, Dr. Yoshihiro Nakatani for providing the P/CAF plasmid and Dr. William Sellers for providing the p300 plasmid. This work was supported by the Hertie Foundation; the Fortune Grant, University of Tuebingen, the NIH R21 NS052640 and the DFG DI 1497/1-1 grants (all granted to Simone Di Giovanni).

1. Teng FY, Tang BL. Axonal regeneration in adult CNS neurons - signaling molecules and pathways. J Neurochem 2006; 96: 1501-1508.

2. Makwana M, Raivich G. Molecular mechanisms in successful peripheral regeneration. FEBS J 2005; 272: 2628-2638.

3. Mellor J. The dynamics of chromatin remodeling at promoters. Mol Cell 2005; 19: 147-157.

4. Reik W. Stability and flexibility of epigenetic gene regulation in mammalian development. Nature 2007; 447: 425-432.

5. Guan JS, Haggarty SJ, Giacometti E, Dannenberg JH, Joseph N, Gao J et al. HDAC2 negatively regulates memory formation and synaptic plasticity. Nature 2009; 459: $55-60$.

6. Thiagalingam S, Cheng $\mathrm{KH}$, Lee $\mathrm{HJ}$, Mineva $\mathrm{N}$, Thiagalingam A, Ponte JF. Histone deacetylases: unique players in shaping the epigenetic histone code. Ann NY Acad Sci 2003: 983: 84-100.

7. Yang XJ, Seto E. HATs and HDACs: from structure, function and regulation to novel strategies for therapy and prevention. Oncogene 2007; 26: 5310-5318.

8. Struhl K. Histone acetylation and transcriptional regulatory mechanisms. Genes Dev 1998; 12: 599-606.

9. Wolffe AP, Guschin D. Review: chromatin structural features and targets that regulate transcription. J Struct Biol 2000; 129: 102-122.

10. Saha RN, Pahan K. HATs and HDACs in neurodegeneration: a tale of disconcerted acetylation homeostasis. Cell Death Differ 2006; 13: 539-550.

11. Schwechter BR, Millet LE, Levin LA. Histone deacetylase inhibition-mediated differentiation of RGC-5 cells and interaction with survival. Invest Ophthalmol Vis Sci 2007; 48: 2845-2857.

12. Siebzehnrubl FA, Buslei R, Eyupoglu IY, Seufert S, Hahnen E, Blumcke I. Histone deacetylase inhibitors increase neuronal differentiation in adult forebrain precursor cells. Exp Brain Res 2007; 176: 672-678.

13. Balasubramaniyan V, Boddeke E, Bakels R, Kust B, Kooistra S, Veneman A et al. Effects of histone deacetylation inhibition on neuronal differentiation of embryonic mouse neural stem cells. Neuroscience 2006; 143: 939-951. 
14. Jiang H, Poirier MA, Liang Y, Pei Z, Weiskittel CE, Smith WW et al. Depletion of CBP is directly linked with cellular toxicity caused by mutant huntingtin. Neurobiol Dis 2006; 23: 543-551.

15. Arany Z, Huang LE, Eckner R, Bhattacharya S, Jiang C, Goldberg MA et al. An essential role for p300/CBP in the cellular response to hypoxia. Proc Natl Acad Sci U S A 1996; 93: 12969-12973.

16. Rouaux C, Jokic N, Mbebi C, Boutillier S, Loeffler JP, Boutillier AL. Critical loss of $\mathrm{CBP} / \mathrm{p} 300$ histone acetylase activity by caspase- 6 during neurodegeneration. EMBO J 2003; 22: 6537-6549.

17. Glozak MA, Sengupta N, Zhang X, Seto E. Acetylation and deacetylation of non-histone proteins. Gene 2005; 363: 15-23.

18. Di Giovanni S, Knights CD, Rao M, Yakovlev A, Beers J, Catania J et al. The tumor suppressor protein p53 is required for neurite outgrowth and axon regeneration. EMBO J 2006; 25: 4084-4096.

19. Tedeschi A, Nguyen T, Puttagunta R, Gaub P, Di Giovanni S. A p53-CBP/p300 transcription module is required for GAP-43 expression, axon outgrowth, and regeneration. Cell Death Differ 2009; 16: 543-554.

20. Yoshida M, Kijima M, Akita M, Beppu T. Potent and specific inhibition of mammalian histone deacetylase both in vivo and in vitro by trichostatin A. J Biol Chem 1990; 265 17174-17179.

21. Blagosklonny MV. Flavopiridol, an inhibitor of transcription: implications, problems and solutions. Cell Cycle (Georgetown, Tex) 2004; 3: 1537-1542.

22. Filbin MT. Myelin-associated inhibitors of axonal regeneration in the adult mammalian CNS. Nat Rev Neurosci 2003; 4: 703-713.

23. Allis CD, Berger SL, Cote J, Dent S, Jenuwien T, Kouzarides T et al. New nomenclature for chromatin-modifying enzymes. Cell 2007; 131: 633-636.

24. Nott A, Watson PM, Robinson JD, Crepaldi L, Riccio A. S-nitrosylation of histone deacetylase 2 induces chromatin remodelling in neurons. Nature 2008; 455 : 411-415.

25. Knights CD, Catania J, Di Giovanni S, Muratoglu S, Perez R, Swartzbeck A et al. Distinct p53 acetylation cassettes differentially influence gene-expression patterns and cell fate. J Cell Biol 2006; 173: 533-544.
26. Freeland K, Boxer LM, Latchman DS. The cyclic AMP response element in the Bcl-2 promoter confers inducibility by hypoxia in neuronal cells. Brain Res 2001; 92: 98-106.

27. Gao Y, Deng K, Hou J, Bryson JB, Barco A, Nikulina E et al. Activated CREB is sufficient to overcome inhibitors in myelin and promote spinal axon regeneration in vivo. Neuron 2004; 44: 609-621

28. Jung $M$, Petrausch $B$, Stuermer $C A$. Axon-regenerating retinal ganglion cells in adult rats synthesize the cell adhesion molecule L1 but not TAG-1 or SC-1. Mol Cell Neurosci 1997; 9: 116-131.

29. Aigner L, Arber S, Kapfhammer JP, Laux T, Schneider C, Botteri F et al. Overexpression of the neural growth-associated protein GAP-43 induces nerve sprouting in the adult nervous system of transgenic mice. Cell 1995; 83: 269-278.

30. Aigner L, Caroni P. Depletion of $43-\mathrm{kD}$ growth-associated protein in primary sensory neurons leads to diminished formation and spreading of growth cones. J Cell Biol 1993; 123: 417-429.

31. Benowitz LI, Perrone-Bizzozero NI, Neve RL, Rodriguez W. GAP-43 as a marker for structural plasticity in the mature CNS. Prog Brain Res 1990; 86: 309-320.

32. Gianola S, Rossi F. GAP-43 overexpression in adult mouse Purkinje cells overrides myelinderived inhibition of neurite growth. Eur J Neurosci 2004; 19: 819-830.

33. Di Giovanni S, De Biase A, Yakovlev A, Finn T, Beers J, Hoffman EP et al. In vivo and in vitro characterization of novel neuronal plasticity factors identified following spinal cord injury. J Biol Chem 2005; 280: 2084-2091.

34. Cai L, Marshall TW, Uetrecht AC, Schafer DA, Bear JE. Coronin 1B coordinates Arp2/3 complex and cofilin activities at the leading edge. Cell 2007; 128: 915-929.

35. Di Giovanni S, Movsesyan V, Ahmed F, Cernak I, Schinelli S, Stoica B et al. Cell cycle inhibition provides neuroprotection and reduces glial proliferation and scar formation after traumatic brain injury. Proc Natl Acad Sci USA 2005; 102: 8333-8338.

36. Schousboe A, Drejer J, Hansen GH, Meier E. Cultured neurons as model systems for biochemical and pharmacological studies on receptors for neurotransmitter amino acids. Dev Neurosci 1985; 7: 252-262.

37. Dohda T, Kaneoka H, Inayoshi Y, Kamihira M, Miyake K, lijima S. Transcriptional coactivators CBP and p300 cooperatively enhance HNF-1alpha-mediated expression of the albumin gene in hepatocytes. J Biochem (Tokyo) 2004; 136: 313-319.

\section{Supplementary Information accompanies the paper on Cell Death and Differentiation website (http://www.nature.com/cdd)}

